\title{
Supratrigeminal Bilaterally Projecting Neurons Maintain Basal Tone and Enable Bilateral Phasic Activation of Jaw-Closing Muscles
}

\author{
Edward Stanek IV, ${ }^{1}$ Erica Rodriguez, ${ }^{1}$ Shengli Zhao, ${ }^{1}$ Bao-Xia Han, ${ }^{1}$ and $\odot$ Fan Wang ${ }^{1,2}$ \\ Departments of ${ }^{1}$ Neurobiology and ${ }^{2}$ Cell Biology, Duke University Medical Center, Durham, North Carolina 27710
}

\begin{abstract}
Anatomical studies have identified brainstem neurons that project bilaterally to left and right oromotor pools, which could potentially mediate bilateral muscle coordination. We use retrograde lentiviruses combined with a split-intein-mediated split-Cre-recombinase system in mice to isolate, characterize, and manipulate a population of neurons projecting to both the left and right jaw-closing trigeminal motoneurons. We find that these bilaterally projecting premotor neurons (BPNs) reside primarily in the supratrigeminal nucleus (SupV) and the parvicellular and intermediate reticular regions dorsal to the facial motor nucleus. These BPNs also project to multiple midbrain and brainstem targets implicated in orofacial sensorimotor control, and consist of a mix of glutamatergic, GABAergic, and glycinergic neurons, which can drive both excitatory and inhibitory inputs to trigeminal motoneurons when optogenetically activated in slice. Silencing BPNs with tetanus toxin light chain (TeNT) increases bilateral masseter activation during chewing, an effect driven by the expression of TeNT in SupV BPNs. Acute unilateral optogenetic inhibition of SupV BPNs identifies a group of tonically active neurons that function to lower masseter muscle tone, whereas unilateral optogenetic activation of SupV BPNs is sufficient to induce bilateral masseter activation both during resting state and during chewing. These results provide evidence for SupV BPNs in tonically modulating jawclosing muscle tone and in mediating bilateral jaw closing.
\end{abstract}

Key words: bilaterally projecting neurons; optogenetics; retrograde lentivirus; split-intein-split-Cre system; supratrigeminal nucleus; trigeminal motor nucleus

\section{Significance Statement}

We developed a method that combines retrograde lentiviruses with the split-intein-split-Cre system in mice to isolate, characterize, and manipulate neurons that project to both left and right jaw-closing motoneurons. We show that these bilaterally projecting premotor neurons (BPNs) reside primarily in the supratrigeminal nucleus and the rostral parvicellular and intermediate reticular nuclei. BPNs consist of both excitatory and inhibitory populations, and also project to multiple brainstem nuclei implicated in orofacial sensorimotor control. Manipulation of the supratrigeminal BPNs during natural jaw-closing behavior reveals a dual role for these neurons in eliciting phasic muscle activation and in maintaining basal muscle tone. The retrograde lentivirus carrying the split-intein-split-Cre system can be applied to study any neurons with bifurcating axons innervating two brain regions.

\section{Introduction}

Muscles of the jaw participate in a variety of essential behaviors, including chewing, drinking, licking, breathing, and speaking,

\footnotetext{
Received March 14, 2016; revised June 3, 2016; accepted June 7, 2016.

Author contributions: E.S.I. and F.W. designed research; E.S.I. and E.R. performed research; E.S.I., E.R., S.Z., and B.-X.H. contributed unpublished reagents/analytic tools; E.S.I. and E.R. analyzed data; E.S.I. and F.W. wrote the paper.

This work was supported by grants from the NIH: R01 (NS077986 to F.W.), and F31 NRSA (DE024003-01 to E.S.). We thank Drs Jeffrey Moore, Hao Zhang, Jun Takatoh, Katsuyasu Sakurai, Richard Mooney, Rebecca Yang, Henry Yin, and Stephen Lisberger for their helpful advice during the process of this work and/or comments on this paper. The authors declare no competing financial interests.

Correspondence should be addressed to Dr Fan Wang, Department of Neurobiology, Box 3209, Duke University Medical Center, Durham, NC 27710. E-mail: fan.wang@duke.edu.
}

and must coordinate both with each other and with other muscles of the tongue and face. Dysfunction of jaw muscle activity contributes to numerous orofacial disorders, including sleep bruxism, orofacial dystonia, temporomandibular joint disorder, and others (Richter and Löscher, 1998; Balasubramaniam and Ram, 2008; T. Kato et al., 2011; Suzuki and Iwata, 2016). In mammals, smooth jaw movements require coordinated activity between left and right jaw muscles, as the mandible is joined at the midline. One simple way to enable such coordination is for premotor neurons to project bilaterally to left and right jaw mo- 
toneurons. Indeed, the supratrigeminal nucleus (SupV) and the parvicellular and intermediate reticular nuclei dorsal to the facial motor nucleus (Rt-MoVII) contain neurons that project axons to left and right trigeminal motor nuclei $(\mathrm{MoV})$, which contain jaw-closing motoneurons (Li et al., 1993; Kamogawa et al., 1994). We recently provided evidence that some of these neurons synapse directly onto jaw-closing masseter motoneurons bilaterally (Stanek et al., 2014).

Previous studies showed that neurons in SupV and Rt-MoVII receive orofacial sensory inputs (Li et al., 1998; Dong et al., 2011), and jaw-closing spindle afferent inputs (Luo et al., 2001); and these neurons respond to pressure on the teeth, passive jaw stretch, and sensory stimuli applied to the tongue (Minkels et al., 1991; Inoue et al., 2002; Takamatsu et al., 2005). Stimulation of the sensory nerve innervating the lower jaw activates SupV and Rt-MoVII neurons leading to inhibition of motoneurons in $\mathrm{MoV}$ and in the central facial motor nucleus (Kamogawa et al., 1994; Minkels et al., 1995). These findings have led to the hypothesis that neurons in SupV and Rt-MoVII mediate the motor inhibition component of jaw pain reflexes.

However, SupV and Rt-MoVII contain not only GABAergic and glycinergic neurons which could mediate motor inhibition, but also glutamatergic neurons (Travers et al., 2005; Paik et al., 2009). Physiology studies have indicated that activation of these regions induces inhibitory and excitatory potentials in trigeminal motoneurons (Ohta and Moriyama, 1986; Castillo et al., 1991). Additionally, SupV and Rt-MoVII neurons are highly interconnected with other premotor neurons surrounding MoV (Ter Horst et al., 1991; Kolta, 1997), and only a subset of SupV neurons respond to orofacial sensory stimuli, suggesting that the roles of neurons in these regions may be more complex $(\mathrm{Mu}-$ rakami et al., 1989). Indeed, many neurons in both of these regions are rhythmically active during rhythmic jaw movements (Yamamoto et al., 1989; Inoue et al., 1992), and may relay rhythm information to jaw motoneurons. It is possible that distinct subpopulations of SupV and Rt-MoVII neurons mediate each of these effects. However, there has been a dearth of studies assessing the role of subpopulations of these neurons in freely moving animals.

We set out to characterize the bilaterally projecting neurons (BPNs) that simultaneously innervate both the left and the right $\mathrm{MoV}$, and investigate how these neurons affect jaw muscle activity in the awake behaving mouse. A major barrier to conducting such studies has been a lack of technology to selectively isolate and manipulate BPNs. Here, we take advantage of an intein-mediated split-Cre system developed in our laboratory (Wang et al., 2012) in combination with retrograde lentiviruses to localize and manipulate BPNs, and identify their projection patterns.

In situ hybridization and channelrhodopsin-2 (ChR2)assisted slice physiology revealed BPNs to be a mixed population of excitatory and inhibitory neurons capable of inducing IPSCs and EPSCs in MoV motoneurons. We permanently silenced BPNs in freely moving mice with tetanus-toxin light chain (TeNT), uncovering a role for SupV BPNs in maintaining an appropriate level of masseter activation during chewing and gnawing behavior. To further investigate this effect, we transiently inhibited or activated SupV BPNs during natural jaw activity using optogenetics, and identified two distinct roles for SupV BPNs in jaw closing movement: enabling phasic bilateral muscle activation and dampening basal masseter muscle tone.

\section{Materials and Methods}

All experimental protocols were approved by the Duke University Institutional Animal Care and Use Committee.

Viruses. FuGB2-coated lentiviruses containing Cre-N (RG-LV-Cre-N) and Cre-C (RG-LV-Cre-C) were produced and concentrated as described previously (Zhang et al., 2015). Briefly, HEK293T cells were transiently transfected with pLenti-hSynapsin-CreN or pLentihSynapsin-CreC together with psPAX2 and FuGB2 using polyethyleneimine "MAX" (PEI MAX) (Polysciences 24765). Forty-eight hours after transfection, the cell culture media was collected, centrifuged at 3000 $\mathrm{rpm}$ for $15 \mathrm{~min}$, and the supernatant was filtered with a $0.45 \mu \mathrm{m}$ filter unit (Millipore, SCHVU01RE). The filtered virus-containing supernatant was loaded into the centrifuge tubes with $3 \mathrm{ml}$ of $20 \%$ sucrose cushion at the bottom, spun at 25,000 rpm with Beckman SW-28 rotor for $2 \mathrm{~h}$ at $4^{\circ} \mathrm{C}$. The pellet was resuspended in an appropriate volume of PBS without calcium and magnesium (PBS, $-\mathrm{Ca} 2+,-\mathrm{Mg} 2+$ ), aliquoted and stored at $-800{ }^{\circ} \mathrm{C}$. The viral titer was estimated to be $1 \times 10^{8}$ infection units per milliliter.

AAV8-hsyn-Flex-GFP and AAV8-hSyn-Flex-TeNT-P2A-GFP viruses were generated and concentrated as described before (Zhang et al., 2015). In brief, HEK293T cells were transiently transfected with pAAV-hSynFlex-GFP or pAAV-hSyn-Flex-TeNT-P2A-GFP, together with AAV serotype plasmid AAV8, and pAd.DELTA F6 using PEI MAX (Polysciences 24765). Seventy-two hours post-transfection, the cell culture media was discarded, the cells were collected in PBS, frozen and thawed three times using a dry-ice/ethanol bath, and centrifuged at $3000 \mathrm{rpm}$ for $30 \mathrm{~min}$. The contaminating DNA in the supernatant was removed by adding benzonase and incubated at $37^{\circ} \mathrm{C}$ for $30 \mathrm{~min}$. The viral crude solution was filtered through a $0.45 \mu \mathrm{m}$ filter (Millipore), and spun at 25,000 rpm for 90 min using a Beckman SW-28 rotor. The pellet was resuspended in $4 \mathrm{ml}$ of PBS, filtered through a $0.45 \mu \mathrm{m}$ filter and a $0.22 \mu \mathrm{m}$ filter sequentially. The viral crude solution was concentrated to 200-250 $\mu$ l using the Amicon Ultra-4 filter unit (100 KDa), washed with $4 \mathrm{ml}$ of PBS once, and concentrated to an appropriate volume. The viral solution was further purified using a $0.22 \mu \mathrm{m}$ Ultrafree-MC centrifugal filter unit (Millipore, UFC30GV0S), aliquots were stored at $-80^{\circ} \mathrm{C}$ until use.

Animals. Adult male and female Ail4 mice (Jackson Laboratories, stock 007914) expressing a Cre-dependent tdTomato protein were used for initial BPN labeling, tracing BPN projections, in situ hybridization experiments ( $n=4$ mice, 2 sections/region/mouse for a total of 8 sections per condition), and were also used as controls for optogenetic experiments $(n=4$ mice). Male and female C57B/L6 mice (Jackson Laboratories, stock 000664) were used for TeNT experimental $[n=5$ mice, 2 electromyogram (EMG) channels/mouse] and control ( $n=4$ mice, 2 EMG channels/mouse) animals. Male and female Ai32 mice expressing a Cre-dependent ChR2 (Jackson Laboratories, stock 024109) were used for ChR2 experimental animals ( $n=5$ mice, 2 EMG channels/mouse) and electrophysiology experiments ( $n=13$ cells from 6 mice). Male Ai35 mice expressing a Cre-dependent archaerhodopsin (Arch; Jackson Laboratories, stock 012735) were used for Arch experimental animals ( $n=5$ mice, 2 EMG channels/mouse).

Stereotaxic injections. Animals were deeply anesthetized with an intraperitoneal injection of ketamine $(100 \mathrm{mg} / \mathrm{kg})$ and xylazine $(10 \mathrm{mg} / \mathrm{kg})$ dissolved in saline. Virus was delivered into the target site using a pulled glass capillary (Warner Instruments) connected to an UltraMicroPump controlled by a SYS-Micro4 Controller (World Precision Instruments). For all mice, $300 \mathrm{nl}$ of RG-LV-Cre-N or $300 \mathrm{nl}$ of RG-LV-Cre-C was injected at $200 \mathrm{nl} / \mathrm{min}$ into the left or right $\mathrm{MoV}$, respectively, of 6- to 12-week-old mice (Fig. 1A). To target MoV bilaterally, mice were mounted in the stereotaxic frame at an angle such that lambda was $\sim 180$ $\mu \mathrm{m}$ ventral to bregma (in practice, $140-240 \mu \mathrm{m}$ ). The skull was thinned around the coordinates of $5.00 \mathrm{~mm}$ caudal to bregma and $1.34 \mathrm{~mm}$ lateral to lambda, and the injection pipette was moved just anterior to the transverse sinus. Here at the dura, the dorsal-ventral coordinate of the pipette was measured and the injection site was calculated as $4.20 \mathrm{~mm}$ below this point. A slight twitch or tremor of the ipsilateral cheek occurred during injection of virus to indicate proper targeting of the MoV. 
A

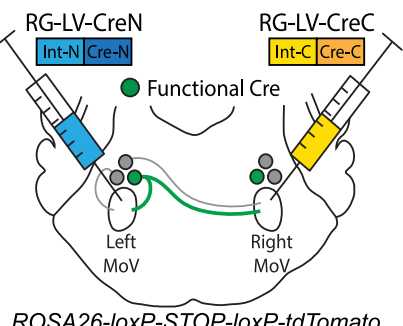

ROSA26-IoxP-STOP-loxP-tdTomato Timeline

Split-Cre injections $^{2}$ weeks Histology
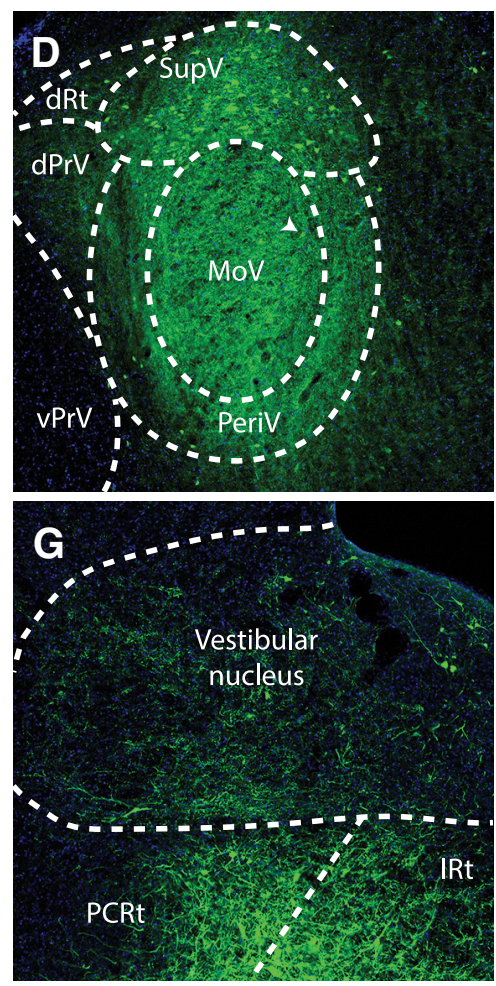
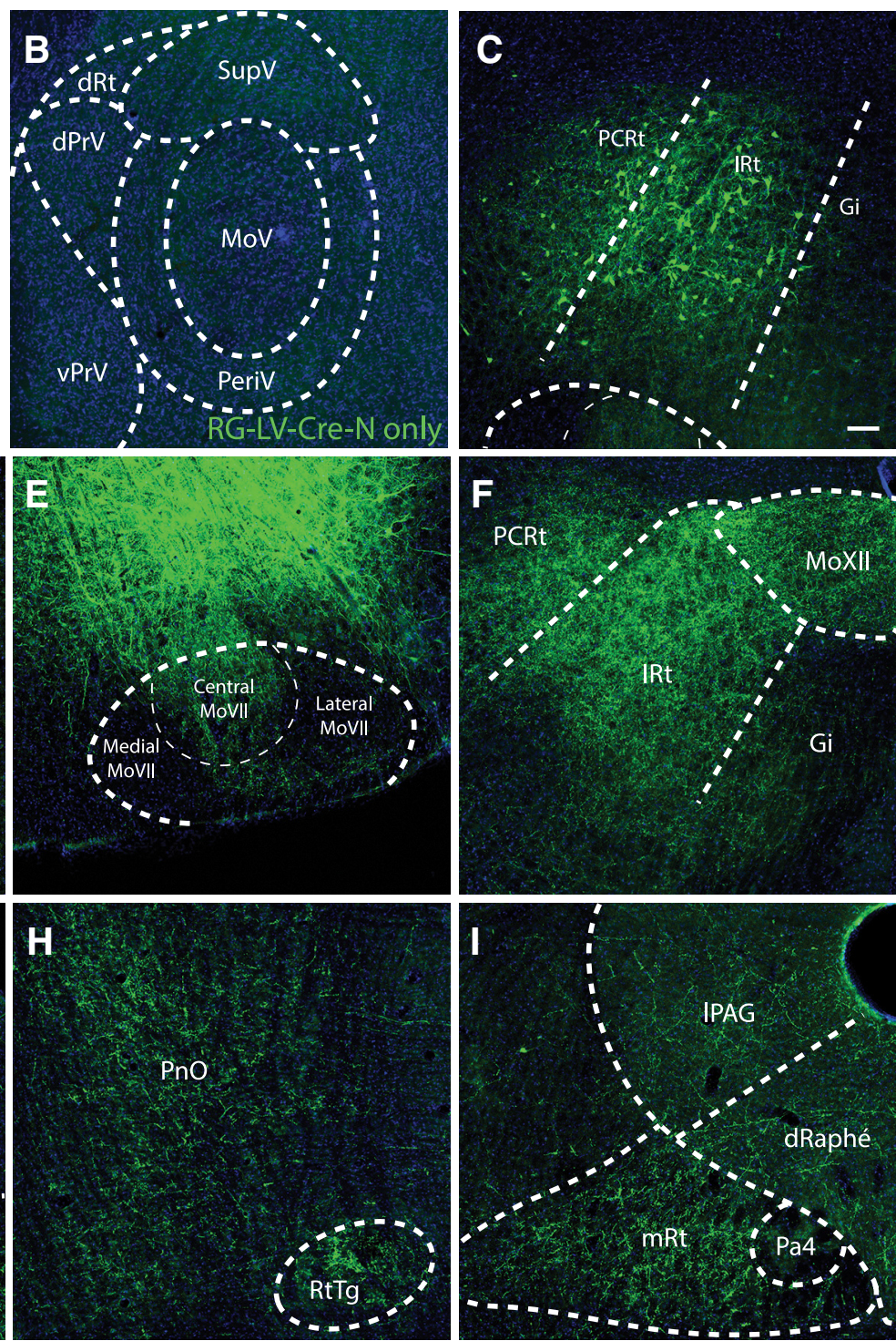

Figure 1. Labeling of BPNs innervating both left and right trigeminal motor nucleus with retrograde lentiviruses expressing split-Cre recombinase. $A$, RG-LV expressing either Cre-N-intein-N or intein-C-Cre-C was injected into the left or right trigeminal MoV, which resulted in the expression of full-length Cre-recombinase selectively in neurons projecting bilaterally to both MoV nuclei. $\boldsymbol{B}$, Injection of only the retrograde lentivirus expressing Cre-N-intein-N into the MoV did not result in any labeled neurons in the brainstem. Similar results were found after injection of only the retrograde lentivirus expressing Cre-C-intein-C into the MoV. C, D, When these viruses were injected into an Ai14 Cre-reporter line, BPNs were identified primarily in the supratrigeminal nucleus dorsal to $\operatorname{MoV}(\boldsymbol{C})$, and the reticular nucleus dorsal to the facial motor nucleus $(\boldsymbol{D})$. $\boldsymbol{E}-\boldsymbol{I}$, Axons of labeled BPNs were also observed projecting bilaterally in the peri-trigeminal region (PeriV; $\boldsymbol{D})$, the central MoVII $(\boldsymbol{E})$, the MoXII and caudal reticular regions (IRt, $\mathrm{PCRt}, \mathrm{Gi} ; \boldsymbol{F})$, the vestibular nucleus $(\boldsymbol{G})$, the oral pontine reticular formation (Pn0) and reticulotegmental nucleus (RtTg; $\boldsymbol{H})$, and the mesencephalic reticular formation (MRF), dorsal raphé (DRN), and lateral periaqueductal gray (IPAG; I). Scale bar, $100 \mu \mathrm{m}$.

The process was repeated on the contralateral side with the complementary virus.

In the TeNT-mediated silencing experiments, AAV-Flex-TeNT-EGFP or AAV-flex-EGFP was injected bilaterally into the parvicellular reticular region at three vertical depths spaced $200 \mu \mathrm{m}$ apart in the same surgery as split-Cre injections. The injection coordinates were relative to the left and right MoV. For example, if the coordinates for $\mathrm{MoV}$ were as follows: posterior to bregma $=\mathrm{B}$; lateral to lambda $=\mathrm{L}$; ventral to dura $=\mathrm{D}$; then the coordinates for AAV injection were $1.0 \mathrm{~mm}$ posterior to B; $60 \mu \mathrm{m}$ medial to $\mathrm{L}$; and 650,500 , and $350 \mu \mathrm{m}$ ventral to D. At each injection site, $150 \mathrm{nl}$ of virus was injected using a syringe pressure injector, resulting in a total of $450 \mathrm{nl}$ of virus injected into each side of the reticular region.

In situ hybridization and immunohistochemistry. All mice were deeply anesthetized and transcardially perfused with $4 \%$ paraformaldehyde (PFA; Sigma-Aldrich) in $1 \times$ PBS (Invitrogen). For tracing studies and in situ hybridization, Ail4 mice injected with the retrograde split-Cre viruses were perfused 2 weeks postinjection. For all other studies, mice were perfused on the last day of data acquisition. Brain samples were then postfixed overnight in $4 \%$ PFA, cryoprotected in a 30\% sucrose (SigmaAldrich) solution in PBS, embedded in optimal cutting temperature compound (Tissue-Tek), and frozen for at least $24 \mathrm{~h}$ at $-80^{\circ} \mathrm{C}$. Immunohistochemistry was performed as previously described (Takatoh et al., 2013). Antibodies used were as follows: rabbit anti-GFP (1:1000, Abcam), rabbit anti-RFP (1:1000, Rockland), GFP anti-rabbit (1:1000, Jackson ImmunoResearch).

Floating section in situ hybridization was performed as follows. For each mouse, six $60 \mu \mathrm{m}$ sections containing Rt-MoVII and six sections containing SupV were collected with a cryostat at $-20^{\circ} \mathrm{C}$ and resuspended in 4\% PFA in DEPC (Sigma-Aldrich) treated double-deionized water in a 24-well plate. Samples were rinsed with $1 \times$ PBS in DEPC water (DEPC PBS), washed with 0.3\% Triton X-100 (Sigma-Aldrich) in DEPC PBS, treated with $5 \mu \mathrm{g} / \mathrm{ml}$ proteinase $\mathrm{K}$ in DEPC PBS, acetylated using a solution of $0.465 \mathrm{~g}$ TEA (Sigma-Aldrich), $28 \mu \mathrm{l} 10 \mathrm{~N} \mathrm{NaOH}, 25 \mathrm{ml}$ DEPC water, $62.6 \mu \mathrm{l}$ acetic anhydride (Sigma-Aldrich), and then rinsed with 
DEPC PBS. Samples were incubated for $1 \mathrm{~h}$ at $60^{\circ} \mathrm{C}$ in hybridization buffer (0.2× SSC, Life Technologies), 10\% blocking reagent (Roche), $50 \%$ formamide, $2 \% \mathrm{~N}$-lauroylsarcosine (NLS; Sigma-Aldrich), $10 \%$ SDS (Sigma-Aldrich), following which probes were prepared as described previously (Hasegawa et al., 2007). GAD1, GAD2, GlyT2, and vGluT2 probes were created as previously described (Takatoh et al., 2013), and GAD1 and GAD2 probes were applied as a mixed probe. The probes were alternated across all sections to ensure that one posterior section and one anterior section from each region was analyzed with each probe type. Samples were incubated with probes overnight in hybridization buffer at $60^{\circ} \mathrm{C}$. The following day, samples were washed with $2 \times$ $\mathrm{SSC} / 50 \%$ formamide $/ 0.1 \% \mathrm{NLS}$ at $60^{\circ} \mathrm{C}$, followed by $2 \times \mathrm{SSC} / 0.1 \% \mathrm{NLS}$, $0.2 \times$ SSC $/ 0.1 \%$ NLS, and finally a wash in Tris-buffered saline containing $0.1 \%$ Triton X-100 (TBST) all at room temperature (RT). Samples were incubated at RT in a 10\% blocking solution in TBST, followed by application of primary antibodies (anti-DIG-AP 1:3500, Roche; rabbit antiRFP 1:200, Rockland) overnight at $4^{\circ} \mathrm{C}$. The following day, samples were washed with TBST, incubated in $100 \mathrm{~mm}$ Tris- $\mathrm{HCl}, \mathrm{pH} 8.0$, incubated in FastRed (Roche) for $90 \mathrm{~min}$, rinsed with TBST, incubated with secondary antibody (GFP-anti-rabbit AlexaFluor 488 1:1000, Jackson ImmunoResearch) for $2 \mathrm{~h}$, stained with DAPI for visualization of nuclei, and mounted onto slides for imaging.

Image acquisition and quantification. Samples were imaged using a Zeiss 700 laser scanning confocal microscope. In situ samples were imaged at $40 \times$ resolution at three $z$-positions. All $z$-positions for each slice were merged into a single image in Adobe Photoshop CS6 for quantification. All other samples were imaged at $20 \times$ resolution. SupV and Rt-MoVII neurons were identified as any neurons within the region dorsal to the MoV or MoVII, respectively, in sections containing these nuclei. All neurons were counted manually. In situ hybridization percentages were calculated within each animal before averaging percentages across animals.

Electrophysiology. Two weeks after viral infection, mice were anesthetized with isofluorane, and transcardially perfused in ice-cold NMDG artificial CSF (NMDG-ACSF; containing 92 mM NMDG, $2.5 \mathrm{~mm} \mathrm{KCl,} 1.2$ mM NaH${ }_{2} \mathrm{PO}_{4}, 30 \mathrm{~mm} \mathrm{NaHCO}, 20 \mathrm{~mm}$ HEPES, 2 mm glucose, $5 \mathrm{~mm}$ sodium ascorbate, $2 \mathrm{~mm}$ thiourea, $3 \mathrm{~mm}$ sodium pyruvate, $10 \mathrm{~mm} \mathrm{MgSO}_{4}$ $0.5 \mathrm{mM} \mathrm{CaCl}_{2}$ ), and bubbled with $5 \% \mathrm{CO}_{2} / 95 \% \mathrm{O}_{2}$. The brain was then extracted and sectioned into $350-\mu \mathrm{m}$-thick sagittal/horizontal slices using a vibratome (VT-1000S, Leica Microsystems) containing ice-cold oxygenated NMDG-ACSF. Coronal sections including the MoV were then bubbled in the same solution at $37^{\circ} \mathrm{C}$ for $8 \mathrm{~min}$, and transferred to bubbled, modified-HEPES ACSF at room temperature $\left(20^{\circ}-25^{\circ} \mathrm{C} ; 92\right.$ mм NaCl, $2.5 \mathrm{~mm} \mathrm{KCl}, 1.2 \mathrm{~mm} \mathrm{NaH} \mathrm{PO}_{4}, 30 \mathrm{~mm} \mathrm{NaHCO}_{3}, 20 \mathrm{~mm}$ HEPES, $2 \mathrm{~mm}$ glucose, $5 \mathrm{~mm}$ sodium ascorbate, $2 \mathrm{~mm}$ thiourea, $3 \mathrm{~mm}$ sodium pyruvate, $2 \mathrm{~mm} \mathrm{MgSO}_{4} 2 \mathrm{mM} \mathrm{CaCl}_{2}$ ) for at least $1 \mathrm{~h}$ before recording. Recordings were performed in a submerged chamber, superfused with continuously bubbled ACSF ( $125 \mathrm{~mm} \mathrm{NaCl}, 2.5 \mathrm{~mm} \mathrm{KCl}, 1.25$ mм NaH${ }_{2} \mathrm{PO}_{4}, 26 \mathrm{~mm} \mathrm{NaHCO}_{3}, 20 \mathrm{~mm}$ glucose, $2 \mathrm{~mm} \mathrm{CaCl}_{2}, 1.3 \mathrm{~mm} \mathrm{Mg}$ $\left.\mathrm{SO}_{4}\right)$ at near-physiological temperature $\left(34 \pm 1^{\circ} \mathrm{C}\right)$. Cells surrounded by axon terminals expressing a virally encoded fluorescent marker (ChR2EYFP) were visualized by infrared differential interference contrast and fluorescence video microscopy (Examiner.D1, Zeiss). Whole-cell voltage-clamp recordings were amplified with Multiclamp 700B (filtered at $2 \mathrm{kHz})$, digitized with Digidata $1440 \mathrm{~A}(5 \mathrm{kHz})$, and recorded using pClamp 10 software (Axon). The patch-clamp electrode (4-6 M $\Omega$ ) was filled with an intracellular solution containing $130 \mathrm{~mm}$ D-gluconic acid, $130 \mathrm{~mm} \mathrm{CsOH}, 5 \mathrm{~mm} \mathrm{NaCl}, 10 \mathrm{~mm}$ HEPES, $12 \mathrm{~mm}$ phosphocreatine, 3 mм MgATP, 0.2 mм Na 2 GTP, 1 mm EGTA. Photostimulation was performed using a $473 \mathrm{~nm}$ LED (CoolLED, pE4000) controlled by pClamp 10 software (Axon). Light intensity was set to be $100 \%$ for generation of spikes in the axon terminals of bilaterally projecting ChR $2+$ neurons with a pulse length of $2 \mathrm{~ms}$. All electrophysiology data were analyzed off-line using Neuromatic package (Think Random) in Igor Pro software (WaveMetrics). Off-line analysis was performed by averaging five traces. Light-evoked EPSC and IPSC peak amplitude, half-width, onset latency, time to peak, rise time, and decay time were analyzed. The onset latency of the light-evoked EPSCs and IPSCs was defined as the time from the onset of the stimulus to the first measurable deflection of the potential from the baseline. Similarly, time to peak was defined as the time from the onset of the stimulus to the peak of the potential. Rise time and decay time were defined as the time between $10 \%$ and $90 \%$ of the rise or decay of the potential, respectively. Two-tailed Student's $t$ tests were performed in GraphPad Prism for all physiology data.

Optic fiber and electromyogram implants. Optic fibers were custommade using stock 0.2-mm-diameter optic fiber cable, and optic cannula glued together with heat-curable glue (ThorLabs). Implant coordinates were obtained using the $\mathrm{A} / \mathrm{P}$ and $\mathrm{M} / \mathrm{L}$ coordinates for the left or right $\mathrm{MoV}$ as described above. The $\mathrm{D} / \mathrm{V}$ coordinate used was $3.50 \mathrm{~mm}$ below the dura. Post-implant, the opening in the skull was filled with bone wax and the optic fiber was fixed to the skull using Gel Control superglue (Home Depot). A depression in the skull above the visual cortex was drilled, and a grounding screw was inserted. A steel grounding wire was wrapped around the grounding screw and soldered to a custom-made seven-contact circuit board (gift from Dr David Kleinfeld, University of California, San Diego, La Jolla, CA).

Following all optic fiber implants, and in TeNT experimental and control animals following injections, EMG electrodes were implanted into the left and right masseter muscles. Twenty-two micrometer EMG electrodes were custom made from coated 0.002 inch tungsten wire (CFW and A-M systems). Each end was stripped $\sim 1 \mu \mathrm{m}$ and one end was bent into a hook. Three electrodes were inserted into the tip of a 27 or 25 guage needle, non-hooked end first. Electrodes were then threaded via the needle beneath the skin at the top of the skull posterior to the eye and into the left and right masseter muscles in each mouse. Each EMG contact was soldered to a custom-made seven-contact circuit board with pins attached, which was further fixed to the skull with superglue. All electronics were coated with dental cement to prevent potential damage from the mouse.

EMG recordings and optogenetic manipulation of SupV bilaterally projecting neurons. At least 1 week postsurgery, mice were briefly anesthetized with vaporized isofluorane. Each mouse was hooked up to a laser via an optic cable (ThorLabs) and a Sirenia acquisition system (Pinnacle Technology). On the first recording day, the optimal pair of EMG electrodes was chosen for each mouse for each muscle. This pair was identified as that with the best signal-to-noise of masseter activity during gnawing or chewing bouts. All subsequent recording sessions collected data from this same pair of electrodes. During optogenetic experiments, a Master-8 pulse stimulator (AMPI) was used to control laser pulses and send a concurrent transistor-to-transistor logic (TTL) pulse to a NI-DAQ system (National Instruments) which translated these pulses to Sirenia Acquisition software (Pinnacle Technology). This software was used to record preprocessed EMG signals and concurrent TTL pulses delivered by the Master- 8 . The EMG sampling frequency was $2 \mathrm{kHz}$ with a high-pass filter of $0.5 \mathrm{~Hz}$ and a low-pass filter of $1 \mathrm{kHz}$.

For BPN optogenetic activation experiments, $20 \mathrm{~ms}$ pulses of a blue laser $(473 \mathrm{~nm})$ were administered at $10 \mathrm{~Hz}$ for $2 \mathrm{~s}$, with a $20 \mathrm{~s}$ interval between stimulation sequences. For BPN inhibition experiments, a $10 \mathrm{~s}$ continuous pulse of a yellow-green laser $(561 \mathrm{~nm})$ was administered with a 40 s interval between pulses. During all experiments, chewing, gnawing, grooming, and various other behavior bouts were manually recorded in a notebook and later transcribed to a Microsoft Excel file for later use.

EMG data analysis. Bilateral EMG raw data were converted from a Sirenia file to an EDF file using Sirenia Acquisition software. TTL data were exported into a TXT file. These data were then imported into MATLAB using edfRead and custom code and extracted into two EMG vectors and a TTL vector to be further processed. Most subsequent data analysis was conducted in either MATLAB using custom code or Excel. Linear regression analyses for comparing slopes were conducted in GraphPad Prism.

Analysis for TeNT-mediated BPN silencing experiments. EMG data were initially filtered between 50 and $500 \mathrm{~Hz}$ using butterworth filters and rectified. For each channel, a threshold for activation was manually recorded. Chewing bouts were extracted from raw EMG traces using manually recorded time ranges. Each time range was assessed for "chewing bouts," where EMG activation in the $5-7 \mathrm{~Hz}$ range (that of reductory chewing) was large and consistent across both channels. This was assessed by bandpass filtering the signal between 5 and $7 \mathrm{~Hz}$ using a but- 
terworth filter, and setting a custom threshold above which standard reductory chewing bouts occurred. A single bout had a minimum duration of $500 \mathrm{~ms}$ to ensure at least three bursts per bout. These bouts were analyzed individually for the average amplitude (defined as local maximum within a single burst above threshold), integrated amplitude (defined as the integral of a burst above threshold), and frequency (bounded between 5 and $7 \mathrm{~Hz}$ ) within a given bout. The mean of these averages was obtained for each day of behavior postinjection. Behavior was assessed $7 \mathrm{~d}$ postinjection and twice per week for the following 2 weeks. Mean amplitude, integrated amplitude, and frequency for each channel for each day was normalized to the mean for that channel $7 \mathrm{~d}$ postinjection. Mean slopes of amplitude or integrated amplitude versus time postinjection were assessed and compared using linear regression in GraphPad Prism.

Analysis for Arch-mediated optogenetic silencing experiments. Raw EMG data were initially segmented into $30 \mathrm{~s}$ samples containing $10 \mathrm{~s}$ of data before each TTL pulse onset and $10 \mathrm{~s}$ of data post-TTL offset. Data were $\mathrm{Z}$-score normalized and a time versus frequency versus power plot was obtained for each sample using the spectrogram function. A $200 \mathrm{~ms}$ window with $50 \%$ overlap was used with an NFFT of 2048. Frequency arrays between the frequencies of 118 and $122 \mathrm{~Hz}$ were averaged within each sample to create a single power versus time vector. These vectors were averaged across each channel to obtain mean power versus time vectors for each channel for each mouse. The means of the first (baseline), second (light-ON), and third (light-OFF) $10 \mathrm{~s}$ of these vectors were extracted for all mice and all values were normalized by subtracting the individual mean baselines. Subsequent normalized values were averaged across all five mice for mean and SEM values. Thresholds for the start of the effect and the end of the effect were defined as the sum of the mean and $1 \mathrm{SD}$ of the mean for either the first $10 \mathrm{~s}$ (start) or trailing $5 \mathrm{~s}$ (end) of mean power arrays for each channel for each mouse. Mean and SEM of these values were calculated across animals.

Muscle tone analysis of chronic silencing and acute activation of bilaterally projecting neurons was assessed in the same manner as above, but with the following alterations. In the chronic silencing experiments, mean power in the 118-122 Hz band across each channel for each mouse were obtained for the entire baseline behavioral session, $7 \mathrm{~d}$ after viral injection, and the entire final behavioral session, $21 \mathrm{~d}$ after viral injection. These values were averaged across channels and across mice to compare power means. In the acute activation experiments, mean power values were assessed during the $2 \mathrm{~s}$ immediately preceding optogenetic stimulation (baseline) and the $2 \mathrm{~s}$ immediately following stimulation responses. These values were normalized by subtracting the mean baseline power and resultant values were averaged across channels across mice to identify a difference in power poststimulation.

Analysis for ChR2-mediated optogenetic activation experiments. For laser-induced burst amplitude analysis, raw EMG was initially segmented into samples containing $6 \mathrm{~s}$ of data: $2 \mathrm{~s}$ before stimulation, the $2 \mathrm{~s}$ of stimulation, and $2 \mathrm{~s}$ poststimulation. Visual inspection confirmed that stimulus-induced peaks were always $\sim 250 \mathrm{~ms}$ post-TTL. Amplitudes of stimulus-induced peaks were assessed as the peak within a $20 \mathrm{~ms}$ window flanking the data point $250 \mathrm{~ms}$ after each TTL pulse. These values were initially normalized by subtracting the mean amplitude of the signal across a given sample. Each normalized amplitude for each peak was then averaged across samples to obtain mean amplitude for each peak. The first peak was analyzed separately from the trailing 19 peaks due to it being much higher than other peaks across all animals. All other peak averages were averaged within each channel, and then values from experimental mice were compared with those obtained from control mice.

To separate trailing peaks occurring during rest, burst, or interburst periods, we initially segregated trials into three groups: rest, chew/gnaw, other activity (including grooming and muscle activations not associated with a clear behavior). Rest trials were defined as containing no natural jaw bursts above threshold either immediately before or following the stimulation period. Chew/gnaw trials and other activity trials were defined as containing some stimulations during an epoch of chewing or gnawing, or other activity, as identified by manually recorded time ranges during behavioral recordings. All trailing peak amplitudes for a given mouse and channel during rest trials were averaged and normal- ized against baseline activity as described above, resulting in average peak above baseline values for each channel for each mouse. These values were averaged to obtain the mean peak above baseline values during rest.

Trailing peaks occurring during chew/gnaw trials were segregated into either burst or interburst peaks via the following method. A Boolean array of each trial was generated such that time points were true if the EMG amplitude was above threshold. Peak ranges for each ChR2induced peak were defined as the peak time point $\pm 10 \mathrm{~ms}$. A ChR2induced peak was categorized as a burst peak if either the time point immediately preceding and/or following the peak range was true in the Boolean array. All other peaks were categorized as interburst peaks. Burst and interburst peaks were then averaged and normalized as described above for rest peaks.

\section{Results}

\section{Labeling of BPNs with retrograde lentiviruses expressing the split-intein-mediated split-Cre recombinase}

We developed a technique combining retrograde lentiviruses with the split-intein-mediated split-Cre recombinase system to label bilaterally projecting premotor neurons. Briefly, pseudotyped lentiviruses packaged with the fusion glycoprotein, FuG-B2 (S. Kato et al., 2011), can infect neurons from axon terminals and retrogradely transport back to the cell soma. We combined this retrograde lentivirus (RG-LV) with a previously developed split-intein-mediated-split-Cre system (Wang et al., 2012; see Materials and Methods). When the Cre-N portion of the split-Cre is expressed in the same cells as the Cre-C portion, the split-intein mediates spontaneous protein-splicing resulting in a full-length intein protein and a full-length, functional Cre recombinase (Wang et al., 2012).

We identified BPNs innervating trigeminal MoV using this RG-LV split-Cre system in an Ail4 mouse line, which expresses tdTomato under the control of Cre (Fig. 1). We first confirmed that neither of the retrograde viruses expressing half-Cre on its own had any recombinase activity by injecting the $\mathrm{MoV}$ with either the retrograde lentivirus expressing Cre-N (RG-LV-Cre-N), or the retrograde lentivirus expressing Cre-C (RG-LV-Cre-C) in Ail4 mice. Two weeks later, we observed no tdTomato signal within the brainstem in either case (Fig. $1 B$, and data not shown).

We then injected RG-LV-Cre-N into the left, and RG-LVCre- $\mathrm{C}$ into the right trigeminal motor nucleus of Ai14 mice (Fig. 1A). Within 2 weeks, we observed labeled BPNs located primarily in the Rt-MoVII, in the peri-trigeminal zone, as well as in the SupV (Fig. 1C,D). BPNs were also distributed bilaterally in the brainstem. Across three Ai14 mice, the proportion of labeled neurons in each mouse on the left side of the brainstem was not significantly different from those on the right side (left: $52.3 \pm$ $2.3 \%$, right: $47.7 \pm 2.3 \%$, two-tailed Student's $t$ test, $t_{(4)}=1.13$, $p=0.32$ ). Furthermore, MoV contains interneurons (McDavid et al., 2006), some of which project bilaterally (Ter Horst et al., 1990), and indeed, we observed sparse labeling of neurons within the boundaries of trigeminal motor nucleus (Fig. 1D, arrow). Other regions less consistently labeled with this viral strategy included the reticular region at the level of the hypoglossal motor nucleus, the medial vestibular nucleus, the spinal trigeminal nucleus interpolaris, the mesencephalic sensory nucleus, and the pontine reticular formation (data not shown). The sporadic labeling in these regions may be caused by variations in injection sites and viral infection.

We examined projection targets of labeled BPNs and found that these neurons also innervate the central MoVII (Fig. 1E) bilaterally, which contains motor neurons of the lips and face, and the hypoglossal motor nucleus (MoXII; Fig. $1 F$ ), which contains motor neurons of the tongue. Extensive axon fibers were 

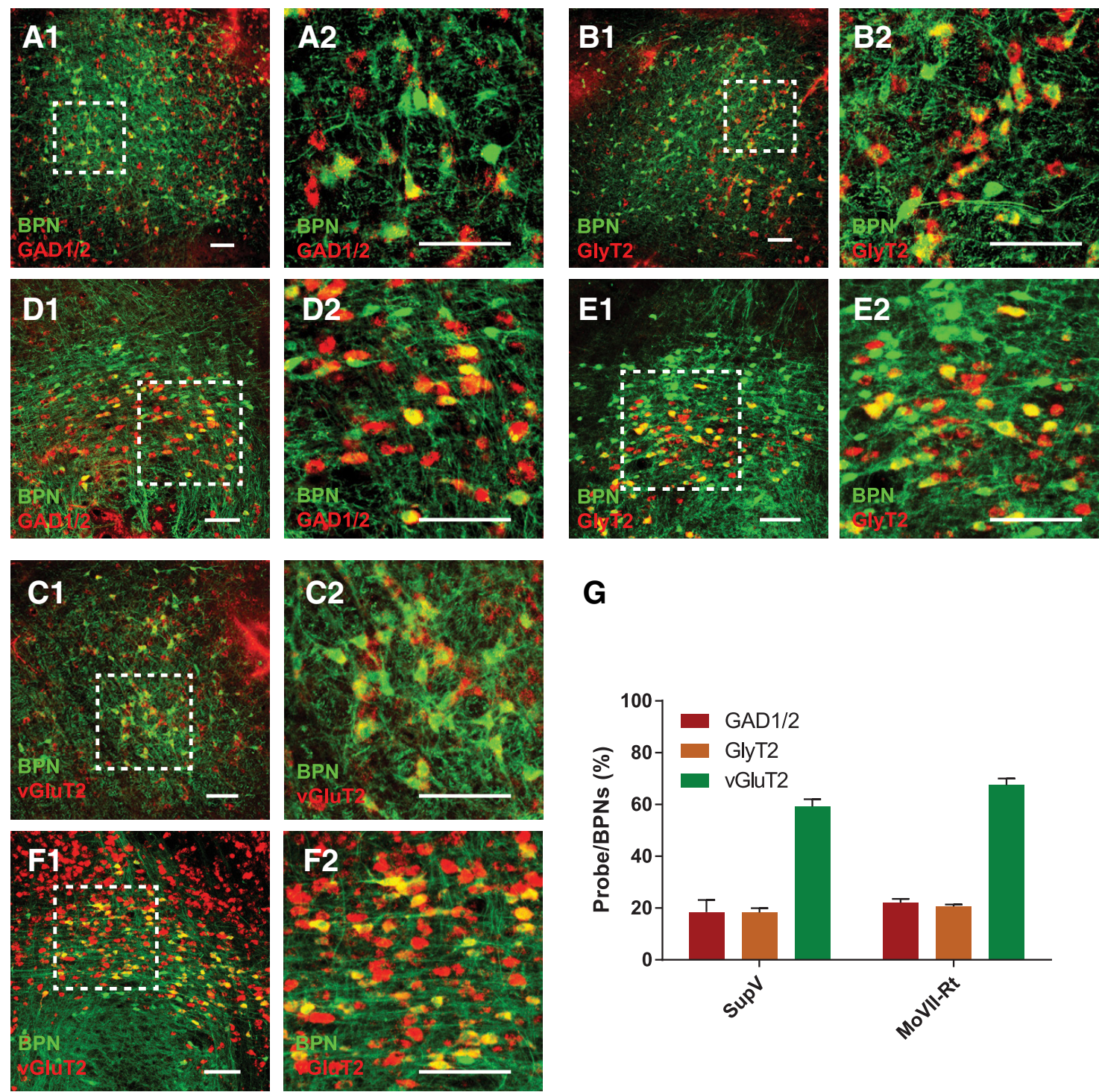

G

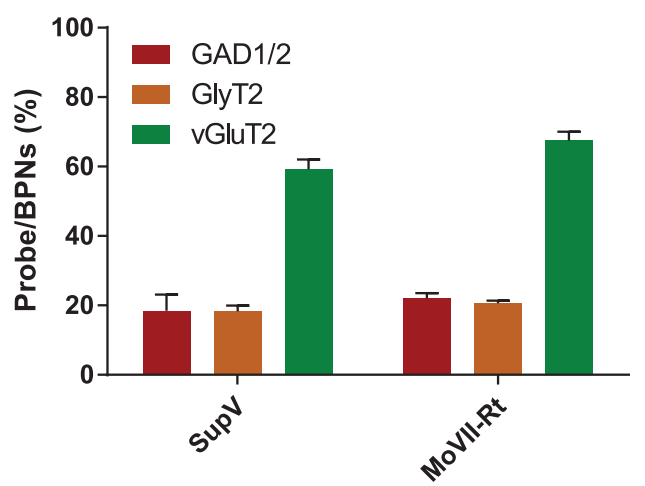

Figure 2. Neurotransmitter phenotypes of SupV and Rt-MoVII BPNs. $\boldsymbol{A}-\boldsymbol{F}$, Representative images of in situ hybridization (red) analyses of BPNs labeled with the split-Cre retrograde lentivirus (green). BPNs in Rt-MoVII and SupV consisted of GABAergic $(\boldsymbol{A}, \boldsymbol{D})$, glycinergic $(\boldsymbol{B}, \boldsymbol{E})$, and glutamatergic neurons $(\boldsymbol{C}, \boldsymbol{F}) . \boldsymbol{G}$, The distributions of neuronal subtypes were similar between Rt-MoVII and SupV. In both cases, the majority of neurons were glutamatergic, while approximately equal proportions of GABAergic and glycinergic neurons were identified. $N=8$ sections of each region per probe from four mice. All values shown are \pm SEM. Scale bar, $100 \mu \mathrm{m}$.

also observed in the caudal medullary and intermediate reticular formation (Fig. $1 F$ ), which is known to contain premotor neurons for jaw and tongue motoneurons (Fay and Norgren, 1997; Kolta et al., 2000; Stanek et al., 2014). Additionally, labeled axons were visible in the vestibular nucleus (Fig. $1 G$ ), the tegmental and pontine reticular formation (Fig. $1 H$ ), the mesencephalic reticular formation, dorsal raphé, and the lateral periaqueductal gray (Fig. 1I).

Neurochemical and electrophysiological analysis reveal both excitatory and inhibitory inputs from BPNs to trigeminal motor neurons

Neurochemical characterization of BPNs

We focused our subsequent experiments on BPNs in Rt-MoVII and SupV as these regions contain the majority of labeled BPNs innervating jaw motoneurons. Both SupV and Rt-MoVII have previously been found to contain glutamatergic, GABAergic, and glycinergic neurons (Travers et al., 2005; Nakamura et al., 2008; Gemba-Nishimura et al., 2010). Some studies using retrograde tracers or dye fills identified inhibitory neurons in SupV that project to both left and right trigeminal motor nuclei (Rokx et al., 1986; Kamogawa et al., 1994), which may simultaneously produce both glycine and GABA (Li et al., 2002). We conducted in situ hybridization experiments in conjunction with immunofluorescence to determine whether BPNs were predominantly inhibitory, excitatory, or a mixture of both (Fig. 2). We began by injecting RG-LV-Cre-N into the left, and RG-LV-Cre-C into the right trigeminal motor nucleus of Ail4 mice. Two weeks later, we probed mRNA expression using either a vGluT2 probe, GlyT2 probe, or mixed GAD1/GAD2 probe along with immunohistochemistry to identify BPNs. SupV and Rt-MoVII BPNs were primarily glutamatergic (SupV, $59.2 \pm 2.8 \%, n=8$ sections, 2 sections/mouse; Rt-MoVII, $67.4 \pm 2.6 \%, n=8$ sections, 2 sections/mouse; Fig. $2 A, D, G)$. Subsets of both SupV and Rt-MoVII BPNs were glycinergic (SupV, $18.3 \pm 1.7 \%, n=8$ sections, 2 sections/mouse; Rt-MoVII, $20.7 \pm 0.7 \%, n=8$ sections, 2 sections/mouse; Fig. $2 B, E, G$ ) or GABAergic (SupV, $18.5 \pm 4.6 \%$, 

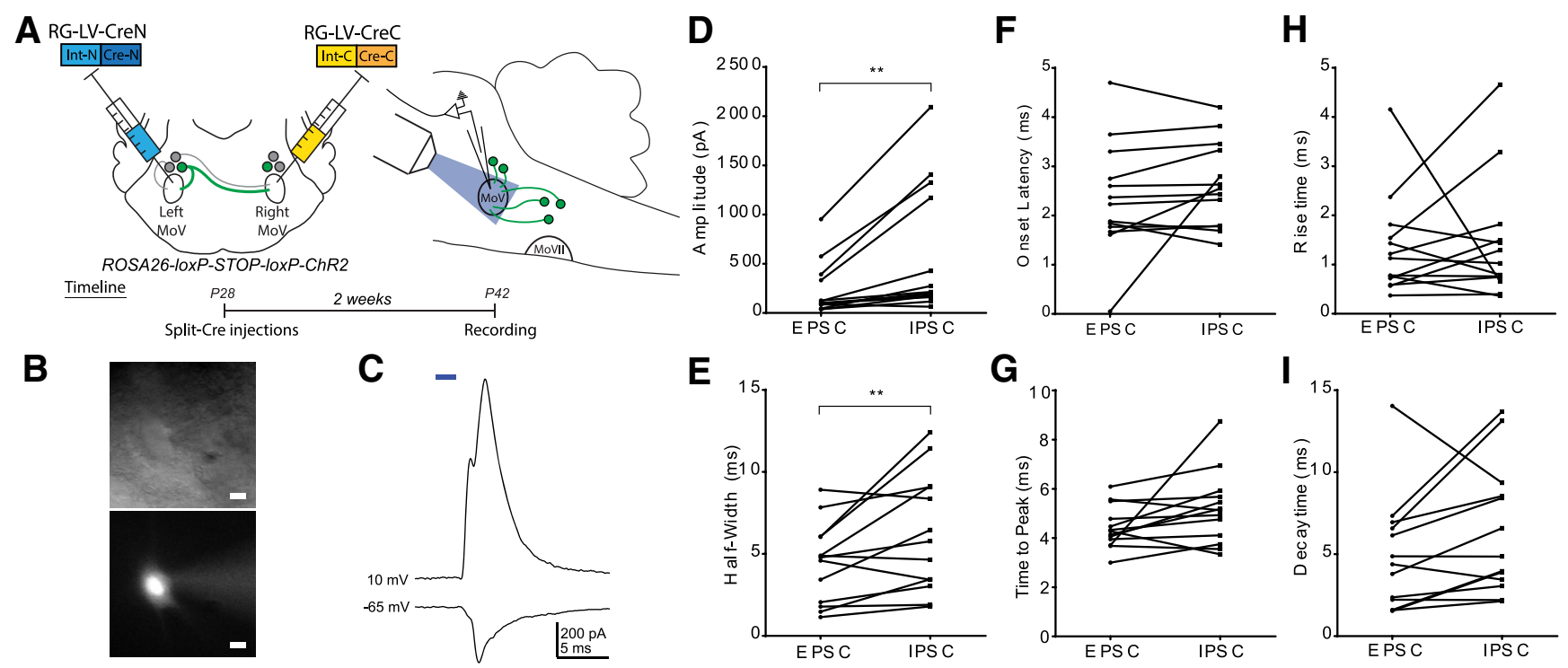

Figure 3. Characterization of trigeminal motoneuron responses to ChR2-assisted stimulation of BPNs in acute slices. $A$, We expressed ChR2 in BPNs by injecting RG-LV-Cre-N and RG-LV-Cre-C into the left and right trigeminal motor nucleus, respectively, in 4-week-old Ai32 mice. Sagittal slices (350 $\mu \mathrm{m})$ were collected for whole-cell patch-clamp physiology. B, Cell fill showing typical MoV motoneuron morphology. Scale bar, $20 \mu \mathrm{m}$. C, Representative traces of excitatory and inhibitory currents from an MoV motoneuron to a 2 ms full-field blue light stimulation of BPNs. MoV motoneuron inhibitory currents showed a significantly larger peak amplitude $(\boldsymbol{D})$ and half-width $(\boldsymbol{E})$ than excitatory currents. $\boldsymbol{F}-\boldsymbol{I}$, There was no significant difference between inhibitory and excitatory currents in onset latency $(\boldsymbol{F})$, time to peak $(\boldsymbol{G})$, rise time $(\boldsymbol{H})$, or decay time $(\boldsymbol{I}) .{ }^{* *} p<0.02 . N=13$ cells from six mice.

$n=8$ sections, 2 sections/mouse; Rt-MoVII, $22.0 \pm 1.5 \%, n=8$ sections, 2 sections/mouse; Fig. $2 C, F, G)$. Thus, BPNs in both SupV and Rt-MoVII consist of a mixed population of glutamatergic, GABAergic, and glycinergic neurons.

\section{Electrophysiological characterization of BPN inputs to} trigeminal motoneurons

We conducted a series of slice physiology experiments to confirm that BPN activation induces both excitatory and inhibitory currents in trigeminal motor neurons (Fig. 3). We expressed Cre in BPNs using the retrograde split-Cre method in Ai32 mice, which allowed Cre-dependent expression of ChR2 (Madisen et al., 2012). Two weeks later, we took acute slices containing trigeminal motor nucleus and conducted patch-clamp recordings on motoneurons in response to blue light stimulation of BPN axons $(n=13$ motoneurons from 6 mice; Fig. $3 A, C)$. Motoneurons showed both an EPSC and IPSC in response to a $2 \mathrm{~ms}$ light pulse, indicating that BPNs can drive both excitatory and inhibitory inputs to jaw motoneurons (Fig. $3 B, D$ ). IPSCs showed a larger peak amplitude (EPSC: $382.1 \mathrm{pA}$, IPSC: $663.6 \mathrm{pA} ; t_{(12)}=3.26$, $p=0.0068$; Fig. $3 D$ ) and half-width (EPSC: $4.453 \mathrm{~ms}$, IPSC: 6.221 $\mathrm{ms} ; t_{(12)}=2.73, p=0.0182$; Fig. $3 E$ ) than EPSCs. Other response characteristics, including the onset latency $\left(t_{(12)}=1.27, p=\right.$ 0.2269 ; Fig. $3 F)$, time to peak $\left(t_{(12)}=1.89, p=0.0835\right.$; Fig. $\left.3 G\right)$, rise time $\left(t_{(12)}=0.26, p=0.7989\right.$; Fig. $\left.3 H\right)$, and decay time $\left(t_{(12)}=1.88, p=0.0848\right.$; Fig. $\left.3 I\right)$ did not differ significantly between EPSCs and IPSCs (all statistical tests performed were two-tailed Student's $t$ tests). Notably, these characteristics are similar to the jaw motoneuron responses induced by electrical stimulation of SupV (Kolta, 1997).

Progressive silencing of subsets of BPNs with TeNT increases bilateral jaw closing amplitude and integrated amplitude dependent on TeNT expression in SupV

As a first step to examine the role of BPNs in regulating bilateral jaw closing movements, we attempted to express TeNT in these neurons (Fig. 4). TeNT cleaves synaptobrevin proteins on synap- tic vesicles and thereby silences the outputs of bilaterally projecting neurons (Yamamoto et al., 2003). To selectively silence BPNs, we injected RG-LV-Cre-N and RG-LV-Cre-C into the left and right trigeminal motor nucleus, respectively, and injected AAVFlex-TeNT-GFP (Zhang et al., 2015) that expressed Credependent TeNT and EGFP into the left and right Rt-MoVII (Fig. $4 A$ ). In a separate group of mice, AAV-Flex-GFP was injected into Rt-MoVII as controls. Because this experimental strategy required four injections into the brainstem, it resulted in varying levels of viral infection in both SupV (16-296 labeled neurons) and Rt-MoVII (506-1943 labeled neurons; Fig. 4B). However, we observed no significant difference between viral infection levels on the left or right side of the brainstem when normalized to total counts of infected neurons in a given animal (left: $49.9 \pm 3.9 \%$; right: $50.1 \pm 3.4 \%$; two-tailed Student's $t$ test, $t_{(8)}=-0.04, p=$ $0.97)$. EMG wires were implanted bilaterally at the time of viral injections. We assessed EMG during chewing and gnawing behaviors beginning 1 week after injection, and twice per week for the next 2 weeks (Fig. 4A).

Despite the fact that the majority of BPNs are glutamatergic neurons, silencing these neurons resulted in a significant increase in masseter muscle activation amplitude, $t_{(18)}=-3.87, p=$ 0.0011 , and increased integrated amplitude, $t_{(18)}=-3.48, p=$ 0.0027 , at $21 \mathrm{~d}$ after injection as compared with $7 \mathrm{~d}$ after injection (two-tailed paired Student's $t$ test; Fig. $4 C, D$ ). We conducted a linear regression to compare changes in integrated amplitude over time between experimental and control mice and found a significant difference in slopes $\left(F_{(1,266)}=56.5345, p<0.0001\right)$. Individual mice, however, showed varying degrees of increases in EMG amplitudes (Fig. 4E). We compared the behavioral effect size with the labeling efficiency to assess whether this variation could be attributed to differences in the number of TeNTexpressing BPNs. When we examined the relationship between TeNT-labeled SupV neurons and the slopes of EMG values, we found that experimental mice with a higher number of TeNTexpressing SupV neurons showed a significant difference in the 
A
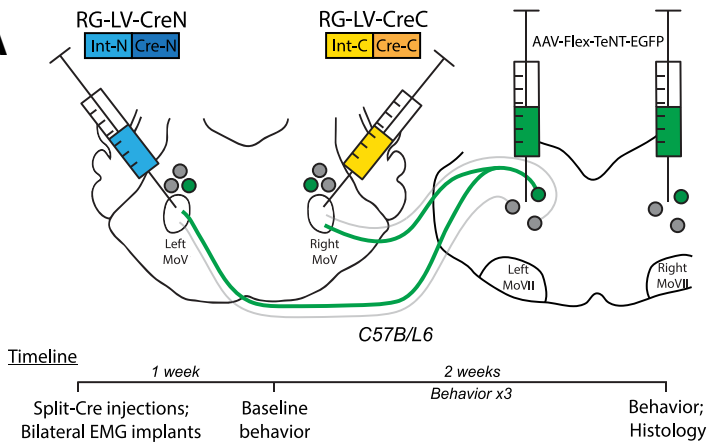

C

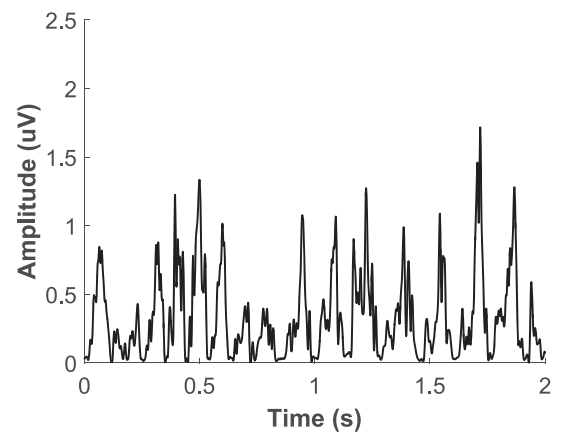

$\mathbf{E}$

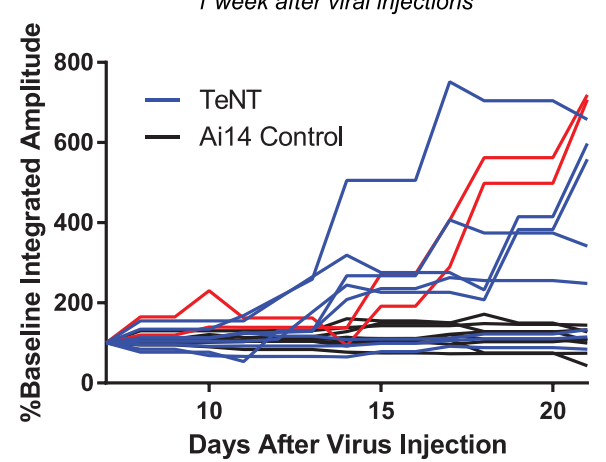

B

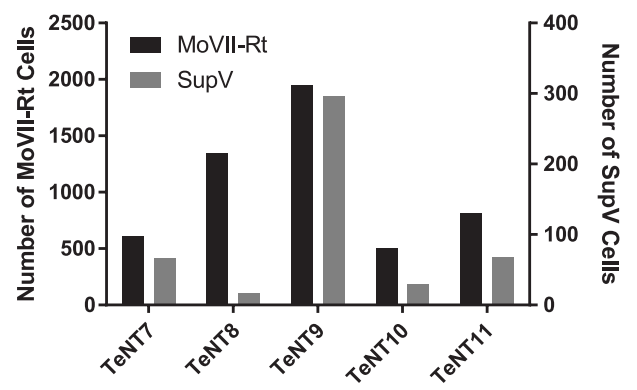

D

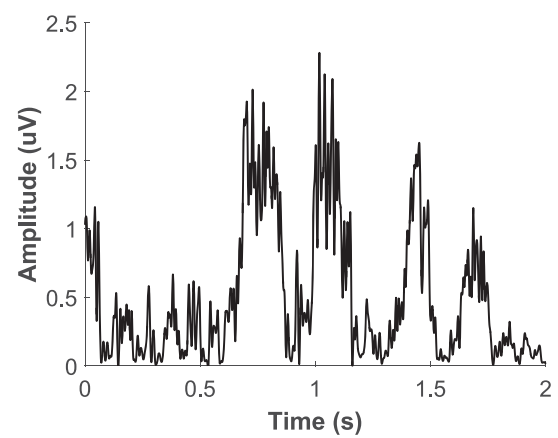

3 weeks after viral injections

$\mathbf{F}$

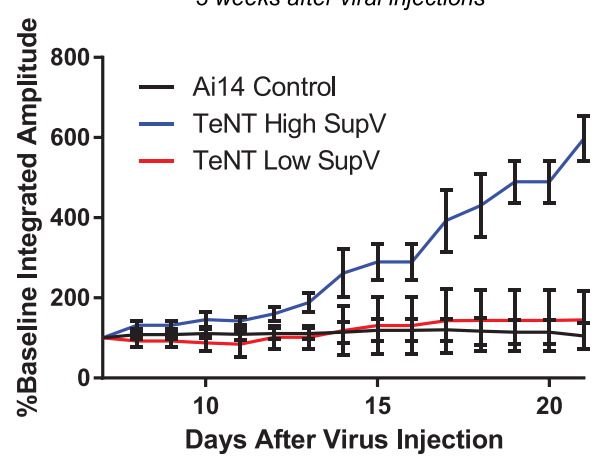

Figure 4. TeNT-mediated chronic silencing of BPN outputs induces bilateral increases in masseter muscle activation amplitude. A, RG-LV-Cre-N and RG-LV-Cre-C were injected into the left and right trigeminal motor nucleus, respectively, to express Cre in bilaterally projecting neurons. Bilateral injections of a Cre-dependent TeNT were targeted for infection of neurons in both Rt-MoVII and SupV. Immediately following injections, EMG implants were inserted into the left and right masseter. $\boldsymbol{B}$, Variations in the numbers of TeNT-infected BPNs in Rt-MoVII and SupV across all five mice. $\boldsymbol{C}-\boldsymbol{E}$, Representative results of masseter EMG during chewing revealed that both burst amplitude and integrated amplitude increased between 1 week $(\boldsymbol{C})$ and 3 weeks $(\boldsymbol{D})$ after viral infection, and this increase occurred to a varying extent across experimental mice ( $\boldsymbol{E}$, red lines indicate left and right masseter integrated amplitude values for TeNT11). $\boldsymbol{F}$, A median split based on the numbers of TeNT-infected SupV BPNs revealed that only mice with higher numbers of TeNT-expressing SupV BPNs had increased masseter amplitude. All values shown are \pm SEM.

slope of measured EMG from controls $\left(F_{(1,206)}=201.809, p<\right.$ $0.0001)$, and from mice with fewer TeNT-labeled SupV BPNs $\left(F_{(1,146)}=71.1288, p<0.0001\right.$; Fig. $\left.4 F\right)$. By contrast, the number of TeNT-labeled Rt-MoVII BPNs showed no significant effects on EMG slopes $\left(F_{(1,146)}=2.84233, p=0.093\right)$. Together, these results indicate that chronically silencing output of SupV BPNs results in an increase in the masseter muscle activation amplitude and integrated amplitude.

Acute optogenetic silencing of SupV-BPNs with archaerhodopsin reveals $\mathrm{BPN}$-mediated tonic inhibition in controlling baseline masseter muscle tone

To investigate how silencing SupV BPNs might increase masseter burst amplitudes during jaw-closing events, we acutely inhibited SupV BPN activity using Arch (Fig. 5). We expressed Cre in BPNs using the retrograde split-Cre method in Ai35 mice, which express Arch under the control of Cre (Madisen et al., 2012). Two weeks later, we implanted an optic fiber unilaterally above the SupV on one side and implanted EMG electrodes into both the left and right masseter (see Materials and Methods; Fig. 5A). At least 1 week later, bilateral masseter muscle activity was recorded in the awake behaving animal during Arch-mediated inhibition of BPNs.

Acute optogenetic inhibition of SupV BPNs induced a significant bilateral increase in baseline muscle tone compared with controls (two-tailed Student's $t$ test, $t_{(16)}=3.02, p=0.008$ ), both during resting (Fig. $5 B$ ), and chewing bouts (Fig. $5 C$ ). We identified this effect as occurring in a high-frequency band centered at $120 \mathrm{~Hz}$ (Fig. 5D). On average, the latency to the onset of increase in EMG power after light-ON was $1.7 \pm 0.3 \mathrm{~s}$, whereas the latency from light-OFF to the decrease in EMG power was $3 \pm 0.4 \mathrm{~s}$. The total duration of elevated muscle tone induced by the $10 \mathrm{~s}$ Arch inhibition was on average $11.1 \pm 0.5 \mathrm{~s}$. Additionally, the muscle tone did not fully return to the prestimulus baseline: there was a persistent albeit small increase in muscle tone bilaterally even after the main effect had disappeared (two-tailed Student's $t$ test, $t_{(16)}=2.59, p=0.020$; Fig. $\left.5 D-F\right)$. This effect slowly diminished over time such that muscle tone returned to baseline by $20 \mathrm{~s}$ after 

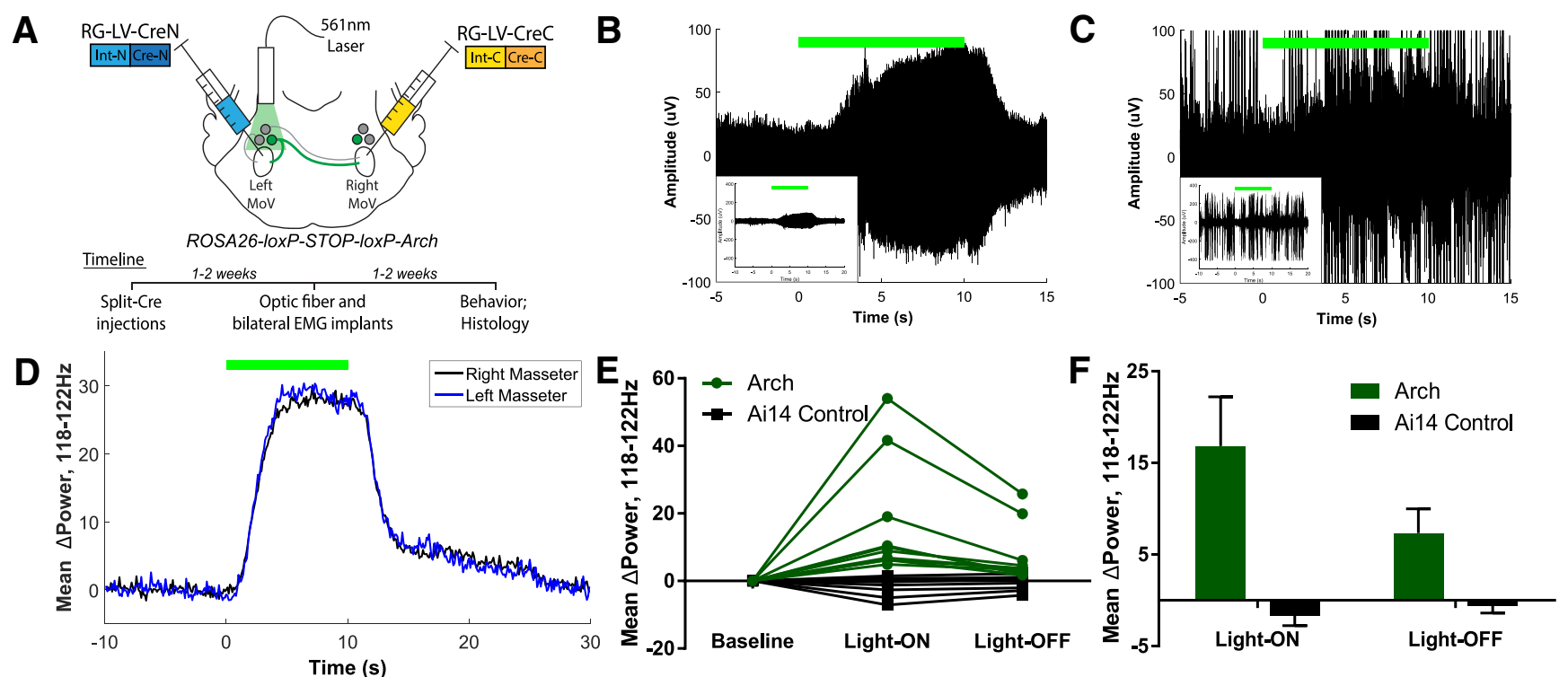

Figure 5. Acute optogenetic inactivation of SupV BPNs reveals a tonic inhibitory function in setting bilateral muscle tone. A, We expressed Arch in BPNs by injecting RG-LV-Cre-N and RG-LV-Cre-C into the left and right trigeminal motor nucleus, respectively, in Ai35 mice. An optic fiber was implanted dorsal to SupV unilaterally, and EMG electrodes were implanted bilaterally into the left and right masseter muscles. $\boldsymbol{B}-\boldsymbol{E}$, Ten second Arch-mediated silencing of SupV BPNs resulted in an increase in masseter muscle tone both during quiet $(\boldsymbol{B})$ and active $(\boldsymbol{C})$ EMG epochs in Ai35 mice $(n=5)$ versus control Ai14 mice $(n=4)$. This effect occurred bilaterally in the $118-122 \mathrm{~Hz}$ band $(\boldsymbol{D})$ in all experimental mice $(\boldsymbol{E})$. $\boldsymbol{F}$, An aftereffect of increased bilateral muscle tone was also observed post-optogenetic silencing of SupV BPNs, which slowly diminished. All values shown are \pm SEM.

light offset (Fig. 5D). Together, these results suggest that inhibition of SupV BPNs produces a net disinhibition of masseter motoneuron activity on both sides, which results in an increase in jaw-closing muscle tone. Furthermore, acute and transient inhibition of SupV BPNs is sufficient to reset baseline muscle tone to a higher level that persists for an extended period of time after the release of inhibition.

\section{ChR2-mediated BPN activation induces short-latency} bilateral jaw-closing twitches time-locked to laser pulses The Arch-mediated silencing experiments suggest a net inhibitory output from SupV BPNs despite that most of these neurons are glutamatergic (Fig. 2G). Yet activation of neurons in SupV was suggested to play a role in transient masseter activation following stimulation of the dental nerve (Kamogawa et al., 1994; Minkels et al., 1995; Takamatsu et al., 2005), and our physiology experiments indicated that BPN activity can produce EPSCs in masseter motoneurons (Fig. 3). To examine the effects of activating SupV BPNs on bilateral jaw closing in the awake behaving animal, we conducted a series of in vivo optogenetic activation experiments with simultaneous bilateral EMG recordings (see Materials and Methods).

To acutely activate BPNs in SupV, we induced ChR2 expression in these neurons by expressing Cre using the retrograde split-Cre method in Ai32 mice (Fig. 6A). One to 2 weeks after viral injection, an optic fiber was unilaterally implanted dorsal to the SupV on one side, and chronic EMG electrodes were implanted into both the left and right masseter muscles (see Materials and Methods). Bilateral masseter muscle activity was recorded while SupV BPNs on one side were activated via $473 \mathrm{~nm}$ laser pulses administered through the optic implant. A previous recording study identified neurons in the peri-trigeminal region, including SupV, as containing neurons that were spontaneously active at firing rates between 3 and $32 \mathrm{~Hz}$ (Bourque and Kolta, 2001). We photo-stimulated BPNs with $20 \mathrm{~ms}$ pulses at $10 \mathrm{~Hz}$ for $2 \mathrm{~s}$ and found a consistent coordinated activation of both the left and right masseter muscles synchronized to the stimulation pulses, with a short delay from onset of the laser $(253 \pm 1 \mathrm{~ms}$ latency; Fig. $6 B$ ). Such activations were visible during both active (chewing, gnawing, or grooming) and quiet (resting) periods of masseter activity (Fig. 6B, C-E; orange bars denote natural jawclosing bursts). Furthermore, photoactivation of BPNs did not prevent initiation of chewing (Fig. $6 C$ ), nor did it interrupt (Fig. $6 D$ ), or prolong chewing (Fig. 6E). The first peak in each stimulation train showed a similar average peak amplitude to that of chewing bursts, whereas all subsequent peaks (trailing peaks) showed average amplitudes of $43.1 \pm 7.2 \%$ of the first peak (Fig. $6 F)$. Normalized light-induced EMG peaks observed in experimental mice were significantly greater than normalized peak EMG values in control mice at similar time delays from light onset (two-tailed Student's $t$ test, first peak: $t_{(16)}=5.37, p<$ 0.0001 ; trailing peaks: $t_{(16)}=3.70, p=0.002$ ). Trailing peaks during the resting state showed significantly higher average amplitudes compared with trailing peaks during the period between naturally occurring masseter EMG bursts (interburst period; two-tailed Student's $t$ test, $t_{(18)}=2.92, p=0.009$; Fig. $6 G$ ). But we did not observe any statistically significant differences in trailing peak amplitudes between those induced during resting state and those occurring during natural bursts (two-tailed Student's $t$ test, $t_{(18)}=1.18, p=0.253$ ), or between those occurring during natural burst and those induced during interburst (two-tailed Student's $t$ test, $t_{(18)}=-1.86, p=0.078$ ). These results indicate that ChR2-mediated activation of BPNs can excite masseter motoneurons in the awake behaving animal.

To assess whether acute activation of SupV BPNs could transiently decrease muscle tone in a manner opposite to that of acutely inhibiting SupV BPNs, we compared the average EMG signal in the $118-122 \mathrm{~Hz}$ frequency band between the $2 \mathrm{~s}$ prestimulation and the $2 \mathrm{~s}$ after stimulation (data not shown). We did not find any significant difference between muscle tone averages prestimulation and poststimulation (two-tailed Student's $t$ test, $t_{(18)}=-0.02, p=0.98$; see Discussion). 


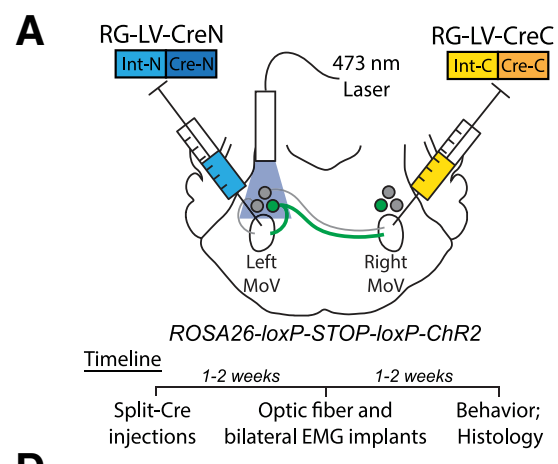

D

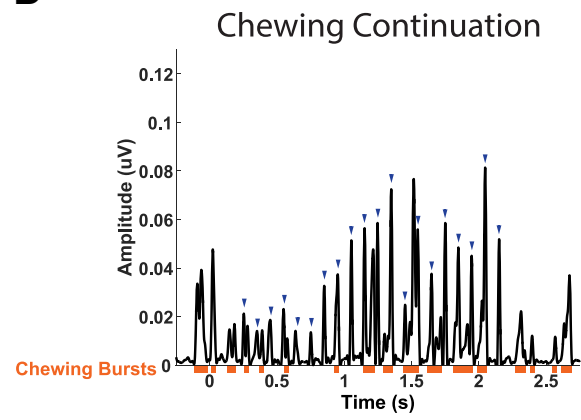

B

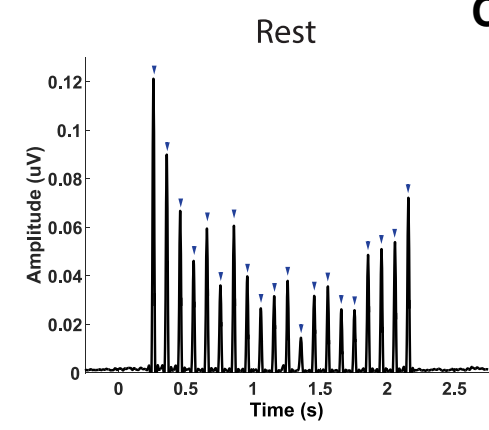

E

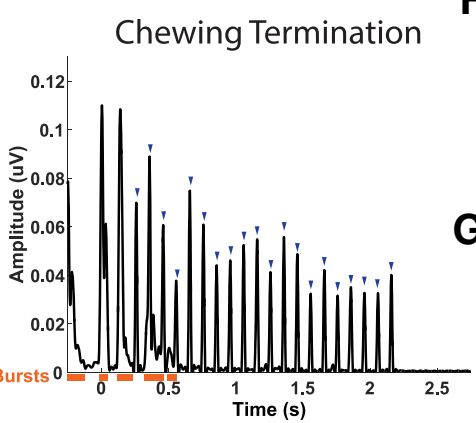

C
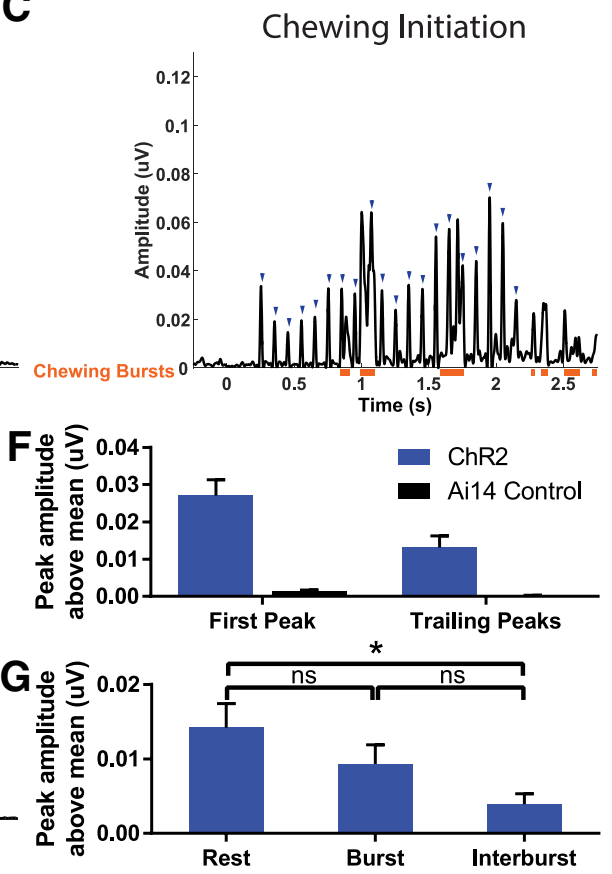

Figure 6. Acute unilateral optogenetic activation of SupV BPNs is sufficient to induce bilateral masseter activation. $A$, We expressed ChR2 in BPNs by injecting RG-LV-Cre-N and RG-LV-Cre-C into the left and right trigeminal motor nucleus, respectively, in Ai32 mice. An optic fiber was implanted dorsal to SupV unilaterally, and EMG electrodes were implanted bilaterally into the left and right masseter muscles. $\boldsymbol{B}-\boldsymbol{E}$, Twenty $20 \mathrm{~ms}$ pulses of blue light administered at a frequency of $10 \mathrm{~Hz}$ induced reliable time-locked bilateral masseter activations (blue arrowheads) in quiet EMG epochs $(\boldsymbol{B})$, and during the initiation $(\boldsymbol{C})$, continuation $(\boldsymbol{D})$, and termination $(\boldsymbol{E})$ of chewing behavior. Orange bars denote spontaneous jaw-closing bursts. All sample traces are from the right masseter of the same mouse. $\boldsymbol{F}$, Light stimulation induced EMG amplitudes in Ai32 mice $(n=5)$ were significantly greater than that observed in control Ai14 mice $(n=4)$. The first peak was approximately twice the size of trailing peaks across all mice. $G$, Average trailing peak amplitudes induced by light during resting state, naturally occurring jaw bursts, or during the interburst period. ${ }^{*} p<0.05$; ns, not statistically significant. All values shown are \pm SEM.

\section{Discussion}

In this study, we developed a method that combines retrograde lentiviruses and the split-intein-split-Cre recombinase system to isolate and manipulate BPNs that innervate both the left and the right trigeminal motor nuclei where masseter motoneurons reside. Our findings are consistent with a simple model in which excitatory and inhibitory SupV BPNs play two distinct roles in jaw closing behavior: excitatory neurons are capable of inducing phasic bilateral activation of masseter muscles that can occur concurrently with natural jaw closing movement, and inhibitory neurons tonically maintain a balanced, low level of masseter muscle tone (see model in Fig. 7).

\section{Characterization of BPNs}

We discovered that BPNs innervating both left and right jaw motoneurons primarily localize to SupV and Rt-MoVII. In situ hybridization experiments identified BPNs as a mixed population of glutamatergic, GABAergic, and glycinergic neurons, with nearly $60 \%$ being glutamatergic. These numbers agree with those of other studies which did not discriminate between bilaterally and unilaterally projecting neurons in SupV and Rt-MoVII (Travers et al., 2005; Paik et al., 2009). ChR2-assisted slice physiology confirmed that activation of BPNs is sufficient to drive both excitatory and inhibitory currents in jaw motoneurons (Fig. 3), in

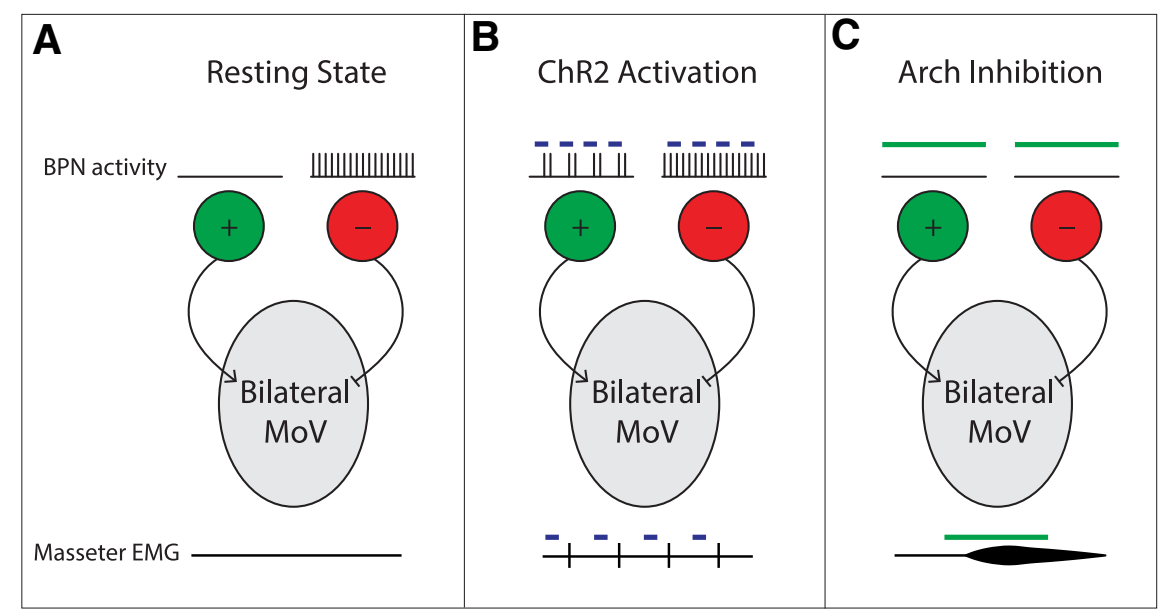

Figure 7. A simple model for SupV BPN functions in regulating masseter motoneuron activity. $\boldsymbol{A}$, During the resting state, excitatory SupV BPNs are inactive while inhibitory neurons are tonically active to maintain a balanced low level masseter muscle tone bilaterally. $\boldsymbol{B}$, During ChR2-mediated activation, excitatory SupV BPNs are activated to induce phasic bilateral masseter EMG responses, whereas inhibitory SupV BPNs remain active to balance muscle tone. C, During Arch-mediated inhibition, excitatory SupV BPNs remain inactive, whereas inhibitory SupV BPNs are silenced, thereby disinhibiting MoV motoneurons resulting in an increase in bilateral masseter muscle tone.

agreement with previous electrical stimulation studies (Takamatsu et al., 2005).

In addition to trigeminal motor nuclei, BPNs also project bilaterally to other orofacial motoneurons, to regions containing orofacial premotor neurons, and to midbrain nuclei implicated in motor functions (Fig. 1). These results indicate that BPNs provide inputs to multiple brainstem circuits and multiple 
groups of orofacial motoneurons. Thus, in addition to regulating jaw motoneurons bilaterally, BPNs likely coordinate multiple motor pools involved in jaw-closing movements, and may coordinate jaw movement with other behaviors.

\section{Functions of Rt-MoVII BPNs}

Chronic silencing of BPNs with a Cre-dependent TeNT induced an increase in masseter muscle amplitude and integrated amplitude, an effect attributable to the functions of SupV-BPNs but not to Rt-MoVII-BPNs (Fig. 4). Although we infected a number of BPNs in Rt-MoVII, we were unable to identify a role for these neurons in jaw-closing behavior. The simplest explanation is that we did not silence sufficient numbers of Rt-MoVII BPNs to observe any effects due to the technical limitation of expressing three different viruses in the same population of neurons spread across a large portion of the brainstem.

Previous studies have implicated neurons located in RtMoVII in relaying the rhythmic signal from central pattern generating circuitry to jaw motoneurons (Yamamoto et al., 1989; Inoue et al., 1992). Because jaw movements are bilaterally synchronized, the BPNs in Rt-MoVII are in an ideal position to relay the rhythmic signal to both sides. However, independent jaw pattern generators are present on both the left and the right side (Ihara et al., 2013), and some Rt-MoVII neurons project to the contralateral Rt-MoVII (Ter Horst et al., 1991). An alternative and perhaps parallel pathway to synchronize the bilateral pattern generators is through contralaterally projecting Rt-MoVII neurons, while ipsilaterally projecting Rt-MoVII premotor neurons relay this rhythmic information to $\mathrm{MoV}$ motoneurons. In this scenario, silencing Rt-MoVII BPNs alone is not sufficient to disrupt bilateral rhythmic activation of the masseter muscles, as parallel circuitry carries out a similar function.

\section{Functions of SupV BPNs}

The chronic silencing experiments uncovered a role of SupV BPNs in maintaining an appropriate level of masseter muscle activation during natural jaw closing behavior. To further investigate this phenomenon, we acutely silenced SupV BPNs by expressing Arch under the control of Cre in these neurons (Fig. 5). We discovered that optogenetically silencing SupV BPNs unilaterally disinhibits masseter motoneurons bilaterally, resulting in bilateral increases in muscle tone. Previous studies have found evidence for a tonic inhibitory GABAergic and glycinergic drive to trigeminal motoneurons (Enomoto et al., 1987; Brooks and Peever, 2008). SupV has been found to contain tonically active neurons (Kolta, 1997). Our results connect these two findings and suggest that the tonically active SupV BPNs produce an inhibitory drive onto masseter motoneurons, likely driven by GABAergic and glycinergic transmission (Figs. 5, 2). Although the simplest explanation is that this drive arises directly from tonically active SupV inhibitory BPNs (Fig. 7C), excitatory SupV $\mathrm{BPNs}$ that project to other midbrain and hindbrain regions (Fig. 1) could alternatively activate these targets to indirectly inhibit MoV motoneurons.

SupV BPNs project to other orofacial motor pools, and thus are positioned to coordinate activity between multiple face, tongue, and jaw muscles. Although we focused on masseter muscle activity, it is conceivable that SupV inhibitory BPNs have a similar effect on muscle tone in other orofacial muscles. Such a system capable of simultaneously inhibiting muscle tone in multiple muscles could be recruited by sleep circuitry to enable the muscle atonia observed during sleep (Werth et al., 2002). Dysfunction of sleep-related muscle tone and activity has been implicated in numerous orofacial disorders, including sleep bruxism, obstructive sleep apnea, rapid eye movement sleep behavior disorder, and cataplexy (Horner, 2000; Boeve, 2010; T. Kato et al., 2011; Burgess and Peever, 2013). Future studies should further unravel the relationship between SupV BPN activity and muscle activity in sleep and sleep-related disorders.

Acute silencing of SupV BPNs resulted in an increase in muscle tone, suggesting that SupV BPNs balance masseter muscle tone against another tonic excitatory input to MoV. However, TeNT-mediated chronic silencing of SupV BPNs did not induced a similar increase in the average EMG response in the $118-122 \mathrm{~Hz}$ range across behavioral sessions (two-tailed Student's $t$ test, $t_{(18}$ $=0.546, p=0.59$ ). Because SupV BPNs are permanently silenced by TeNT, it is possible that compensatory plasticity occurs in such chronic conditions to reduce the tonic excitatory input revealed by our acute inhibition experiments. Mesencephalic proprioceptive afferent neurons have been shown to provide excitatory monosynaptic input to jaw motoneurons (Lingenhöhl and Friauf, 1991), and thus are the likely source of this tonic excitation. Interestingly, mesencephalic proprioceptive neurons also provide input to neurons in SupV (Luo et al., 2001). It is thus possible that these proprioceptive neurons send excitatory inputs to both masseter motoneurons and to SupV inhibitory BPNs to balance the muscle tone.

Despite that inhibition of SupV BPNs increased muscle tone, acute activation of these neurons using ChR2 elicited stereotyped bilateral masseter activations time-locked to the stimulus pulse onset (Fig. 6). These activations occurred on top of natural muscle bursts during chewing indicating that excitatory SupV BPNs are capable of increasing masseter activity both during resting and active epochs. These neurons are likely inactive under normal conditions, therefore optogenetic silencing of these normally inactive neurons did not produce an obvious effect (Fig. 7). In response to biting on a hard piece of food, the force of jaw closing increases correspondingly (Liu et al., 1993). It is possible that the excitatory SupV BPNs receive sensory inputs from teeth and other oral cavity afferents, and together with mesencephalic spindle afferents increase activation of jaw-closing motoneurons to facilitate chewing of hard food.

We noted that the amplitude of the first ChR2-induced muscle burst was on average twice the amplitude induced by subsequent light pulses, suggesting that ChR2 stimulation of excitatory SupV BPNs recruits inhibitory circuitry bilaterally to limit subsequent muscle activation. Among the trailing peaks, responses occurring during the period between natural masseter EMG bursts were even further inhibited from those occurring during the resting state (Fig. $6 G$ ). Previous work indicates that trigeminal motoneurons receive rhythmic glycinergic input during the interburst period of chewing (Inoue et al., 1994). Together, these data suggest that inhibitory circuitry recruited by ChR2 activation of SupV BPNs may be distinct from rhythmic glycinergic inhibition present during rhythmic jaw movements, and that these two sources of inhibition may combine to reduce ChR2induced trailing peak amplitude during the interburst period.

Due to the positioning of SupV directly dorsal to trigeminal motor nucleus, unilateral optogenetic manipulation likely affected other BPN afferent axons penetrating the nucleus on the same side, including axons from Rt-MoVII bilaterally. However, unilateral optogenetic manipulations affect bilateral masseter activity with the same time delay and to the same extent in all mice, consistent with the idea that such manipulations mainly affect cell bodies of bilaterally projecting SupV neurons. Additionally, optic fibers were positioned dorsal to SupV, which would maxi- 
mally affect SupV BPNs. Thus, although we cannot rule out the contribution of Rt-MoVII neurons to the observed optogenetic effects, we believe the majority of the effects can be attributed to SupV BPNs, consistent with our findings in the TeNT silencing experiments.

In summary, this study has identified two distinct roles for SupV BPNs in regulating jaw closing in the freely behaving mouse (Fig. 7). It will be important for future studies to investigate how SupV BPN activity is controlled by sensory and top-down inputs, and how this activity affects jaw, tongue, and facial muscle output in the awake state versus during sleep and sleep disorders. Finally, although we focused our study on BPNs innervating jaw motoneurons, the retrograde split-Cre technology can be applied to identify any neurons with bifurcating axons that simultaneously innervate two brain regions, providing a unique strategy to isolate and manipulate neurons based on their connectivity rather than cell type.

\section{References}

Balasubramaniam R, Ram S (2008) Orofacial movement disorders. Oral Maxillofac Surg Clin North Am 20:273-285, vii. Medline

Boeve BF (2010) REM sleep behavior disorder. Ann N Y Acad Sci 1184: 15-54. CrossRef Medline

Bourque MJ, Kolta A (2001) Properties and interconnections of trigeminal interneurons of the lateral pontine reticular formation in the rat. J Neurophysiol 86:2583-2596. Medline

Brooks PL, Peever JH (2008) Glycinergic and GABA(A)-mediated inhibition of somatic motoneurons does not mediate rapid eye movement sleep motor atonia. J Neurosci 28:3535-3545. CrossRef Medline

Burgess CR, Peever JH (2013) A noradrenergic mechanism functions to couple motor behavior with arousal state. Curr Biol 23:1719-1725. CrossRef Medline

Castillo P, Pedroarena C, Chase MH, Morales FR (1991) Strychnine blockade of the non-reciprocal inhibition of trigeminal motoneurons induced by stimulation of the parvocellular reticular formation. Brain Res 567: 346-349. CrossRef Medline

Dong Y, Li J, Zhang F, Li Y (2011) Nociceptive afferents to the premotor neurons that send axons simultaneously to the facial and hypoglossal motoneurons by means of axon collaterals. PLoS One 6:e25615. CrossRef Medline

Enomoto S, Katakura N, Sunada T, Katayama T, Hirose Y, Ishiwata Y, Nakamura Y (1987) Cortically induced masticatory rhythm in masseter motoneurons after blocking inhibition by strychnine and tetanus toxin. Neurosci Res 4:396-412. CrossRef Medline

Fay RA, Norgren R (1997) Identification of rat brainstem multisynaptic connections to the oral motor nuclei using pseudorabies virus: I. Masticatory muscle motor systems. Brain Res Brain Res Rev 25:255-275. CrossRef Medline

Gemba-Nishimura A, Inoue T, Nakamura S, Nakayama K, Mochizuki A, Shintani S, Yoshimura S (2010) Properties of synaptic transmission from the reticular formation dorsal to the facial nucleus to trigeminal motoneurons during early postnatal development in rats. Neuroscience 166:1008-1022. CrossRef Medline

Hasegawa H, Abbott S, Han BX, Qi Y, Wang F (2007) Analyzing somatosensory axon projections with the sensory neuron-specific Advillin gene. J Neurosci 27:14404-14414. CrossRef Medline

Horner RL (2000) Impact of brainstem sleep mechanisms on pharyngeal motor control. Respir Physiol 119:113-121. CrossRef Medline

Ihara Y, Nakayama K, Nakamura S, Mochizuki A, Takahashi K, Inoue T (2013) Coordination of NMDA-induced rhythmic activity in the trigeminal and hypoglossal nerves of neonatal mice in vitro. Neurosci Res 75: 138-149. CrossRef Medline

Inoue M, Nozawa-Inoue K, Donga R, Yamada Y (2002) Convergence of selected inputs from sensory afferents to trigeminal premotor neurons with possible projections to masseter motoneurons in the rabbit. Brain Res 957:183-191. CrossRef Medline

Inoue T, Masuda Y, Nagashima T, Yoshikawa K, Morimoto T (1992) Properties of rhythmically active reticular neurons around the trigeminal motor nucleus during fictive mastication in the rat. Neurosci Res 14:275-294. CrossRef Medline
Inoue T, Chandler SH, Goldberg LJ (1994) Neuropharmacological mechanisms underlying rhythmical discharge in trigeminal interneurons during fictive mastication. J Neurophysiol 71:2061-2073. Medline

Kamogawa H, Manabe K, Kondo M, Naito K (1994) Supra- and juxtatrigeminal inhibitory premotor neurons with bifurcating axons projecting to masseter motoneurons on both sides. Brain Res 639:85-92. CrossRef Medline

Kato S, Kobayashi K, Inoue K, Kuramochi M, Okada T, Yaginuma H, Morimoto K, Shimada T, Takada M, Kobayashi K (2011) A lentiviral strategy for highly efficient retrograde gene transfer by pseudotyping with fusion envelope glycoprotein. Hum Gene Ther 22:197-206. CrossRef Medline

Kato T, Masuda Y, Yoshida A, Morimoto T (2011) Masseter EMG activity during sleep and sleep bruxism. Arch Ital Biol 149:478-491. CrossRef Medline

Kolta A (1997) In vitro investigation of synaptic relations between interneurons surrounding the trigeminal motor nucleus and masseteric motoneurons. J Neurophysiol 78:1720-1725. Medline

Kolta A, Westberg KG, Lund JP (2000) Identification of brainstem interneurons projecting to the trigeminal motor nucleus and adjacent structures in the rabbit. J Chem Neuroanat 19:175-195. CrossRef Medline

Li JL, Kaneko T, Nomura S, Mizuno N (1998) Projections from the caudal spinal trigeminal nucleus to commissural interneurons in the supratrigeminal region: an electron microscope study in the rat. Neurosci Lett 254:57-60. CrossRef Medline

Li YQ, Takada M, Mizuno N (1993) Identification of premotor interneurons which project bilaterally to the trigeminal motor, facial or hypoglossal nuclei: a fluorescent retrograde double-labeling study in the rat. Brain Res 611:160-164. CrossRef Medline

Li YQ, Tao FS, Okamoto K, Nomura S, Kaneko T, Mizuno N (2002) The supratrigeminal region of the rat sends GABA/glycine-cocontaining axon terminals to the motor trigeminal nucleus on the contralateral side. Neurosci Lett 330:13-16. CrossRef Medline

Lingenhöhl K, Friauf E (1991) Sensory neurons and motoneurons of the jaw-closing reflex pathway in rats: a combined morphological and physiological study using the intracellular horseradish peroxidase technique. Exp Brain Res 83:385-396. Medline

Liu ZJ, Masuda Y, Inoue T, Fuchihata H, Sumida A, Takada K, Morimoto T (1993) Coordination of cortically induced rhythmic jaw and tongue movements in the rabbit. J Neurophysiol 69:569-584. Medline

Luo P, Moritani M, Dessem D (2001) Jaw-muscle spindle afferent pathways to the trigeminal motor nucleus in the rat. J Comp Neurol 435:341-353. CrossRef Medline

Madisen L, Mao T, Koch H, Zhuo JM, Berenyi A, Fujisawa S, Hsu YW, Garcia AJ 3rd, Gu X, Zanella S, Kidney J, Gu H, Mao Y, Hooks BM, Boyden ES, Buzsáki G, Ramirez JM, Jones AR, Svoboda K, Han X, et al. (2012) A toolbox of Cre-dependent optogenetic transgenic mice for light-induced activation and silencing. Nat Neurosci 15:793-802. CrossRef Medline

McDavid S, Lund JP, Auclair F, Kolta A (2006) Morphological and immunohistochemical characterization of interneurons within the rat trigeminal motor nucleus. Neuroscience 139:1049-1059. CrossRef Medline

Minkels RF, Jüch PJ, Ter Horst GJ, Van Willigen JD (1991) Projections of the parvocellular reticular formation to the contralateral mesencephalic trigeminal nucleus in the rat. Brain Res 547:13-21. CrossRef Medline

Minkels RF, Jüch PJ, van Willigen JD (1995) Interneurones of the supratrigeminal area mediating reflex inhibition of trigeminal and facial motorneurones in the rat. Arch Oral Biol 40:275-284. CrossRef Medline

Murakami T, Miyata H, Ishizuka K, Suzuki Y (1989) Responses of premotor neurons for the trigeminal motor nucleus to stimulation of the cortical masticatory area and natural stimulation of the oral-facial area (in Japanese). Shigaku 77:898-903. Medline

Nakamura S, Inoue T, Nakajima K, Moritani M, Nakayama K, Tokita K, Yoshida A, Maki K (2008) Synaptic transmission from the supratrigeminal region to jaw-closing and jaw-opening motoneurons in developing rats. J Neurophysiol 100:1885-1896. CrossRef Medline

Ohta M, Moriyama Y (1986) Supratrigeminal neurons mediate the shortest, disynaptic pathway from the central amygdaloid nucleus to the contralateral trigeminal motoneurons in the rat. Comp Biochem Physiol A Comp Physiol 83:633-641. CrossRef Medline

Paik SK, Lee HJ, Choi MK, Cho YS, Park MJ, Moritani M, Yoshida A, Kim YS, Bae YC (2009) Ultrastructural analysis of glutamate-, GABA-, and glycine-immunopositive boutons from supratrigeminal premo- 
toneurons in the rat trigeminal motor nucleus. J Neurosci Res 87: 1115-1122. CrossRef Medline

Richter A, Löscher W (1998) Pathology of idiopathic dystonia: findings from genetic animal models. Prog Neurobiol 54:633-677. CrossRef Medline

Rokx JT, van Willigen JD, Jüch PJ (1986) Bilateral brainstem connections of the rat supratrigeminal region. Acta Anat 127:16-21. CrossRef Medline

Stanek E 4th, Cheng S, Takatoh J, Han BX, Wang F (2014) Monosynaptic premotor circuit tracing reveals neural substrates for oro-motor coordination. Elife 3:e02511. CrossRef Medline

Suzuki A, Iwata J (2016) Mouse genetic models for temporomandibular joint development and disorders. Oral Dis 22:33-38. CrossRef Medline

Takamatsu J, Inoue T, Tsuruoka M, Suganuma T, Furuya R, Kawawa T (2005) Involvement of reticular neurons located dorsal to the facial nucleus in activation of the jaw-closing muscle in rats. Brain Res 1055: 93-102. CrossRef Medline

Takatoh J, Nelson A, Zhou X, Bolton MM, Ehlers MD, Arenkiel BR, Mooney $\mathrm{R}$, Wang F (2013) New modules are added to vibrissal premotor circuitry with the emergence of exploratory whisking. Neuron 77:346-360. CrossRef Medline

Ter Horst GJ, Copray JC, Van Willigen JD, Liem RS (1990) Contralateral projections of cells in the motor trigeminal nucleus of the rat. Neurosci Lett 113:260-266. CrossRef Medline

Ter Horst GJ, Copray JC, Liem RS, Van Willigen JD (1991) Projections from the rostral parvocellular reticular formation to pontine and medullary nuclei in the rat: involvement in autonomic regulation and orofacial motor control. Neuroscience 40:735-758. CrossRef Medline

Travers JB, Yoo JE, Chandran R, Herman K, Travers SP (2005) Neurotransmitter phenotypes of intermediate zone reticular formation projections to the motor trigeminal and hypoglossal nuclei in the rat. J Comp Neurol 488:28-47. CrossRef Medline

Wang P, Chen T, Sakurai K, Han BX, He Z, Feng G, Wang F (2012) Intersectional Cre driver lines generated using split-intein mediated split-Cre reconstitution. Sci Rep 2:497. CrossRef Medline

Werth E, Achermann P, Borbély AA (2002) Selective REM sleep deprivation during daytime: II. Muscle atonia in non-REM sleep. Am J Physiol Regul Integr Comp Physiol 283:R527-R532. CrossRef Medline

Yamamoto M, Wada N, Kitabatake Y, Watanabe D, Anzai M, Yokoyama M, Teranishi Y, Nakanishi S (2003) Reversible suppression of glutamatergic neurotransmission of cerebellar granule cells in vivo by genetically manipulated expression of tetanus neurotoxin light chain. J Neurosci 23:6759-6767. Medline

Yamamoto T, Matsuo R, Kiyomitsu Y, Kitamura R (1989) Sensory and motor responses of trigeminal and reticular neurons during ingestive behavior in rats. Exp Brain Res 76:386-400. Medline

Zhang Y, Zhao S, Rodriguez E, Takatoh J, Han BX, Zhou X, Wang F (2015) Identifying local and descending inputs for primary sensory neurons. J Clin Invest 125:3782-3794. CrossRef Medline 